\title{
Integrated analysis of the aging brain transcriptome and proteome in tauopathy
}

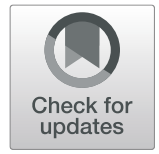

Carl Grant Mangleburg ${ }^{1,2+}$, Timothy Wu ${ }^{1,2+}$, Hari K. Yalamanchili ${ }^{1 \dagger}$, Caiwei Guo ${ }^{3}$, Yi-Chen Hsieh$^{1}$, Duc M. Duong ${ }^{4}$, Eric B. Dammer ${ }^{4}$, Philip L. De Jager ${ }^{5,6}$, Nicholas T. Seyfried ${ }^{4,7}$, Zhandong Lii ${ }^{8,9}$ and Joshua M. Shulman ${ }^{1,3,9,10^{*}}$ (i)

\begin{abstract}
Background: Tau neurofibrillary tangle pathology characterizes Alzheimer's disease and other neurodegenerative tauopathies. Brain gene expression profiles can reveal mechanisms; however, few studies have systematically examined both the transcriptome and proteome or differentiated Tau- versus age-dependent changes.

Methods: Paired, longitudinal RNA-sequencing and mass-spectrometry were performed in a Drosophila model of tauopathy, based on pan-neuronal expression of human wildtype Tau (Tau ${ }^{\text {WT }}$ ) or a mutant form causing frontotemporal dementia ( $\mathrm{Tau}^{\mathrm{R} 406 \mathrm{~W}}$ ). Tau-induced, differentially expressed transcripts and proteins were examined cross-sectionally or using linear regression and adjusting for age. Hierarchical clustering was performed to highlight network perturbations, and we examined overlaps with human brain gene expression profiles in tauopathy.

Results: Tau ${ }^{W T}$ induced 1514 and 213 differentially expressed transcripts and proteins, respectively. Tau ${ }^{\text {R406W }}$ had a substantially greater impact, causing changes in 5494 transcripts and 697 proteins. There was a $~ 70 \%$ overlap between age- and Tau-induced changes and our analyses reveal pervasive bi-directional interactions. Strikingly, 42\% of Tau-induced transcripts were discordant in the proteome, showing opposite direction of change. Tau-responsive gene expression networks strongly implicate innate immune activation. Cross-species analyses pinpoint human brain gene perturbations specifically triggered by Tau pathology and/or aging, and further differentiate between disease amplifying and protective changes.

Conclusions: Our results comprise a powerful, cross-species functional genomics resource for tauopathy, revealing Tau-mediated disruption of gene expression, including dynamic, age-dependent interactions between the brain transcriptome and proteome.
\end{abstract}

Keywords: MAPT, Tau, Alzheimer's disease, Transcriptome, Proteome, Inflammation, Innate immunity

\section{Background}

The Microtubule Associated Protein Tau (MAPT/Tau) aggregates to form neurofibrillary tangle pathology in Alzheimer's disease $(\mathrm{AD})$ and other neurodegenerative tauopathies

\footnotetext{
* Correspondence: Joshua.Shulman@bcm.edu

${ }^{+}$Carl Grant Mangleburg, Timothy Wu and Hari K. Yalamanchili are co-first authors

'Department of Molecular and Human Genetics, Baylor College of Medicine, Houston, TX 77030, USA

${ }^{3}$ Department of Neuroscience, Baylor College of Medicine, Houston, TX 77030, USA

Full list of author information is available at the end of the article
}

characterized by progressive cognitive and/or motor disability, including progressive supranuclear palsy (PSP), corticobasal degeneration, chronic traumatic encephalopathy, and certain forms of frontotemporal dementia (FTD) [1, 2]. Rare mutations in the $M A P T$ gene cause familial FTD, which is also characterized by prominent neurofibrillary tangle deposition [3-5]. Based on this genetic evidence, along with results from cellular and animal models [6, 7], Tau is a critical mediator of age-related neurodegeneration and a causal link among this diverse group of neurologic disorders. While the precise mechanisms of Tau-induced neuronal injury

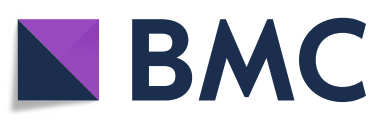

(- The Author(s). 2020 Open Access This article is licensed under a Creative Commons Attribution 4.0 International License, which permits use, sharing, adaptation, distribution and reproduction in any medium or format, as long as you give appropriate credit to the original author(s) and the source, provide a link to the Creative Commons licence, and indicate if changes were made. The images or other third party material in this article are included in the article's Creative Commons licence, unless indicated otherwise in a credit line to the material. If material is not included in the article's Creative Commons licence and your intended use is not permitted by statutory regulation or exceeds the permitted use, you will need to obtain permission directly from the copyright holder. To view a copy of this licence, visit http://creativecommons.org/licenses/by/4.0/. The Creative Commons Public Domain Dedication waiver (http://creativecommons.org/publicdomain/zero/1.0/) applies to the data made available in this article, unless otherwise stated in a credit line to the data. 
remain incompletely defined, progressive synaptic dysfunction and neuronal loss likely arises from a cascade of cellular derangements, including oxidative- and immune-mediated injury, altered proteostasis, and aberrant transcription and translation $[6,8]$.

RNA-sequencing (RNA-seq) makes possible comprehensive gene expression profiling of postmortem human brain tissue in $\mathrm{AD}$ and other tauopathies, providing a systems-level view of transcriptome perturbations accompanying neurodegeneration [9-12]. However, interpretation of differential gene expression analysis is hindered by a number of potential limitations. One major challenge arises from the recognition that the pathologic cascade in $\mathrm{AD}$ and related disorders initiates decades prior to onset of clinical manifestations [13, 14], whereas human brain expression profiles can only be generated cross-sectionally at the time of death. Indeed, it is essential to reconstruct the longitudinal, agingdependent time-course of molecular derangements in order to pinpoint the earliest opportunities for intervention and to develop more effective biomarkers. Second, most brains from older persons with dementia show mixed pathologies at autopsy [15]. Therefore, it can be difficult to differentiate Tau-induced specific expression changes from those caused by other lesions (e.g. amyloid plaques, infarcts, etc.) or brain aging more generally. Third, among associated gene expression changes, it is important to identify those perturbations that are truly primary and therefore causal, rather than simply a consequence of disease. Lastly, emerging evidence suggests that transcription and translation are frequently discordant [16], making it important to consider both mRNA and protein changes to resolve many disease-associated expression signatures. While recent advances in massspectrometry permit deep surveys of protein expression, few studies have systematically profiled both the brain transcriptome and proteome in $\mathrm{AD}$ and related tauopathies $[17,18]$.

By contrast with studies of human postmortem tissue, transgenic animal models of tauopathy readily permit controlled experimental manipulations to (i) define agedependent changes, (ii) isolate the specific impact of Tau, and (iii) definitively establish causation. For example, RNA-seq in mouse transgenic models of tauopathy have highlighted early upregulation of inflammatory processes and downregulation of synaptic function genes preceding behavioral phenotypes, and suggest Tauspecific impact on microglial and neuronal function [19-21]. Expression of human MAPT in the nervous system of the fruit fly, Drosophila melanogaster, recapitulates many key features of tauopathies, including misfolded/hyperphosphorylated Tau, age-dependent synaptic dysfunction and neuronal loss, and reduced survival [6, 22]. Importantly, Drosophila permits high- throughput genetic manipulation, and these models have been successfully deployed for enhancer-suppressor screens [23-25]. The results highlight many promising modifiers of Tau-mediated neurodegeneration, including genes that overlap with human $\mathrm{AD}$ susceptibility loci [26-28]. Prior gene expression studies in fly tauopathy models have been limited by incomplete coverage [29] or cross-sectional design [30], and none have coupled analyses of both transcripts and proteins. We have analyzed longitudinal, paired transcriptome and proteomes from control flies and following pan-neuronal expression of either wildtype or mutant forms of human Tau. We identify Tau-induced patterns of differential expression that are robust to adjustment for aging, and we integrate our results with complementary expression profiles from human brains affected by tauopathy and known genetic modifiers of Tau neurotoxicity.

\section{Methods}

Drosophila stocks and husbandry

$U_{A S-T a u^{W T}}$ and UAS-Tau ${ }^{R 406 W}$ transgenic flies, as previously described in Wittmann et al. 2001, were crossed with the pan-neuronal expression driver elav-GAL4 to generate experimental animals with the genotype elav-GAL4/+;UAS-Tau ${ }^{W T} /+$ or elav-GAL4/ $Y ; U A S-T_{a u}^{W T} /+\quad\left(\right.$ elav $\left.>T^{W a}{ }^{W T}\right)$ and elav-GAL4/+; $U A S-T a u^{R 406 W} /+$ or elav-GAL4/Y;UAS-Tau ${ }^{R 406 W} /+$ $\left(\right.$ elav $\left.>T^{2} u^{R 406 W}\right)$, respectively. These flies express the human Tau 0N4R isoform (383 amino acids). For control animals, we used the genotypes: elavGAL4/+ and elav-GAL4/Y. All flies were raised on standard molasses-based Drosophila media at $25^{\circ} \mathrm{C}$ with ambient light conditions, and aged to 1-, 10-, or 20-days following eclosion. We confirmed expression of Tau at similar levels in elav $>\operatorname{Tau}^{W T}$ and elav $>T^{T a u^{R 406 W}}$ flies using western blot analysis, as previously described [31] using the following antibodies: rabbit anti-Tau (1:5000, Dako); rabbit antiGAPDH (1:5000, GeneTex) and HRP-conjugated anti-rabbit (1:10000, Santa Cruz).

\section{Drosophila RNA-sequencing data}

The Drosophila RNA-sequencing (RNA-seq) dataset analyzed for this work was generated as part of another study, where it is described in detail [31]. Briefly, for elav > Tau and elav controls, animals were evaluated at 1-, 10-, or 20-days. To avoid possible batch effects, experimental and control genotypes used for each comparison $\left(\mathrm{Tau}^{\mathrm{WT}}\right.$ and $\left.\mathrm{Tau}^{\mathrm{R406W}}\right)$ were sequenced together, such that 2 separate control datasets were generated (control 1 and control 2, respectively) for the $\mathrm{Tau}^{\mathrm{WT}}$ and $\mathrm{Tau}^{\mathrm{R} 406 \mathrm{~W}}$ RNA-seq analyses. Triplicate samples $(n=3)$ were used for all genotypes and time points, except for the elav control genotype used for the 
comparison with $\operatorname{Tau}^{R 406 W}$ (control 2), for which duplicate samples were used $(n=2)$ :

\begin{tabular}{lllll}
\hline Exp & Replicates (n) & Ctrl & Replicates (n) & Age (d) \\
Tau $^{W T}$ & 3 & elav & 3 & $1,10,20$ \\
Tau $^{\text {R406W }}$ & 3 & elav & 2 & $1,10,20$ \\
\hline
\end{tabular}

Thus, the transcriptome study included a total of 33 samples. Total RNA was extracted from approximately 100 adult fly heads (for each genotype/age/sample), equally divided between males and females. Sequencing was performed on the Illumina HiSeq with 100 bp pairedend reads. Gene expression values from each sample were quantified as the number of reads mapped (to a specific gene) by setting --quantMode to GeneCounts in STAR 2.5.3a [32]. Genes with an average read count $<50$ across all samples in the comparison were excluded, resulting in count data on 17,104 transcripts across all samples in the final dataset. Unsupervised clustering of samples was assessed by UMAP using DESeq2 depth normalized read counts as described in [33].

\section{Protein extraction and mass-spectrometry}

For proteomics, the identical genotypes (elav $>\operatorname{Tau}^{W T}$, elav $>T_{a}{ }^{R 406 W}$, and elav), time points (1-, 10-, or 20days), and conditions were evaluated as for the RNA-seq analyses. Triplicate samples $(n=3)$ were used for all genotypes and timepoints, and a single control series (control 3) was used since all samples were processed together (27 total samples). Drosophila proteomics were performed according to previously published protocols [18]. Each replicate (40 fly heads of 1:1 male/female ratio per sample) was homogenized in $500 \mathrm{uL}$ of urea lysis buffer (8 M urea, $100 \mathrm{mM} \mathrm{NaHPO}$, pH 8.5), including $5 \mu \mathrm{L}$ (100x stock) HALT protease and phosphatase inhibitor cocktail (Pierce). Protein supernatants were transferred to $1.5 \mathrm{~mL}$ Eppendorf tubes and sonicated (Sonic Dismembrator, Fisher Scientific) 3 times for $5 \mathrm{~s}$ with $15 \mathrm{~s}$ intervals of rest at $30 \%$ amplitude to disrupt nucleic acids and subsequently vortexed. Protein concentration was determined by the bicinchoninic acid (BCA) method, and samples were frozen in aliquots at $80^{\circ} \mathrm{C}$. Each brain homogenate was analyzed by SDSPAGE to assess for protein integrity. Protein homogenates $(150 \mu \mathrm{g})$ were diluted with $50 \mathrm{mM} \mathrm{NH}_{4} \mathrm{HCO}_{3}$ to a final concentration of less than $2 \mathrm{M}$ urea and then treated with $1 \mathrm{mM}$ dithiothreitol (DTT) at $25^{\circ} \mathrm{C}$ for 30 min, followed by $5 \mathrm{mM}$ iodoacetimide (IAA) at $25^{\circ} \mathrm{C}$ for $30 \mathrm{~min}$ in the dark. Protein was digested with 1:100 (w/ w) lysyl endopeptidase (Wako) at $25^{\circ} \mathrm{C}$ for $2 \mathrm{~h}$ and further digested overnight with 1:50 (w/w) trypsin (Promega) at $25^{\circ} \mathrm{C}$. Resulting peptides were desalted with a Sep-Pak C18 column (Waters), dried under vacuum, and
$2 \mu \mathrm{g}$ was resuspended in peptide loading buffer $(0.1 \%$ formic acid, $0.03 \%$ trifluoroacetic acid, $1 \%$ acetonitrile). Peptide mixtures were separated on a self-packed C18 (1.9 $\mu \mathrm{m}$ Dr. Maisch, Germany) fused silica column (25 $\mathrm{cm} \times 75 \mu \mathrm{M}$ internal diameter (ID); New Objective, Woburn, MA) by a NanoAcquity UHPLC (Waters, Milford, FA) and monitored on a Q-Exactive Plus mass spectrometer (ThermoFisher Scientific, San Jose, CA). Elution was performed over a 120-min gradient at a rate of $400 \mathrm{~nL} / \mathrm{min}$ with buffer B ranging from 3 to $80 \%$ (buffer A: $0.1 \%$ formic acid and $5 \%$ DMSO in water, buffer B: $0.1 \%$ formic and $5 \%$ DMSO in acetonitrile). The mass spectrometer cycle was programmed to collect one full MS scan followed by 10 data dependent MS/MS scans. The MS scans $(300-1800 \mathrm{~m} / \mathrm{z}$ range, $1,000,000 \mathrm{AGC}$, $150 \mathrm{~ms}$ maximum ion time) were collected at a resolution of 70,000 at m/z 200 in profile mode and the MS/ MS spectra ( $2 \mathrm{~m} / \mathrm{z}$ isolation width, $25 \%$ collision energy, 100,000 AGC target, $50 \mathrm{~ms}$ maximum ion time) were acquired at a resolution of 17,500 at $\mathrm{m} / \mathrm{z} 200$. Dynamic exclusion was set to exclude previous sequenced precursor ions for $30 \mathrm{~s}$ within a $10 \mathrm{ppm}$ window. Precursor ions with +1 , and +6 or higher charge states were excluded from sequencing.

Raw data for all samples was analyzed using MaxQuant v1.5.2.8 with Thermo Foundation 2.0 for file reading capability. The search engine Andromeda, integrated into MaxQuant, was used to build and search a Uniprot fly database consisting of 13,704 target sequences, plus 245 contaminant proteins from the common repository of adventitious proteins (cRAP) built into MaxQuant. Methionine oxidation (+ 15.9949 $\mathrm{Da})$, asparagine and glutamine deamidation $(+0.9840$ $\mathrm{Da})$, and protein $\mathrm{N}$-terminal acetylation $(+42.0106 \mathrm{Da})$ were variable modifications (up to 5 allowed per peptide); cysteine was assigned a fixed carbamidomethyl modification (+57.0215 Da). Only fully tryptic peptides were considered with up to 2 miscleavages in the database search. A precursor mass tolerance of \pm 20 ppm was applied prior to mass accuracy calibration and $\pm 4.5 \mathrm{ppm}$ after internal MaxQuant calibration. Other search settings included a maximum peptide mass of $6000 \mathrm{Da}$, a minimum peptide length of 6 residues, $0.05 \mathrm{Da}$ tolerance for high resolution MS/MS scans. Co-fragmented peptide search was enabled to deconvolute multiplex spectra. The false discovery rate (FDR) for peptide spectral matches, proteins, and site decoy fraction were all set to 1\%. Quantification settings were as follows: re-quantify with a second peak finding attempt after protein identification has completed; match MS1 peaks between runs; a 0.7 min retention time match window was used after an alignment function was found with a 20-min RT search space. The quantitation method only considered razor plus unique peptides for protein level quantitation. 
Quantitation of proteins was performed using LFQ (labelfree quantification) intensities given by MaxQuant. The match-between-run (MBR) method was applied as part of the MaxQuant pipeline [34]. Overall, only 18\% (12,100 of 65,589 ) of all measured LFQs were derived from MBR. Among the final dataset of 2843 proteins, MBR identification was applicable, on average, in 4 out of 27 total samples total. The full list of parameters used for MaxQuant are available as parameters.txt accompanying the public release (see Availability of Data and Materials).

A total of $n=3584$ unique proteins were initially identified by label-free LC-MS/MS across our proteomic dataset, and we additionally required that each protein have complete, non-missing values among triplicate samples in at least 1 experimental group, resulting in a filtered dataset of $n=2843$ protein isoforms. After collapsing and averaging protein values with multiple isoforms, our final dataset included $n=2723$ unique proteins. Among the 27 samples included in our proteomic survey, we detected a mean of 2267 unique proteins per sample (range $=1214-2547)$ (Additional file 2: Table S1). Among Tau ${ }^{\mathrm{WT}}$ and controls (18 samples), 1479 proteins were consistently detected. Among $\mathrm{Tau}^{\mathrm{R} 406 \mathrm{~W}}$ and controls (18 samples, same controls as for $\mathrm{Tau}^{\mathrm{WT}}$ ), there were 1007 proteins consistently detected. 985 proteins were consistently detected across all 27 samples. For the 351 possible pairwise combinations among samples, there was a mean sample-to-sample overlap of 2015 proteins. Missing proteomic LFQ values were imputed on a per sample basis as previously described in [35]. Missing values were imputed by drawing from a Gaussian distribution simulating expression near the LFQ detection limit, a down-shift of 1.8 standard deviations from the median sample expression. For quality assurance, we tabulated for each protein the number of replicate samples with complete data (non-imputed), broken down by genotype and age (Additional file 2: Table S11). Abundance data for UniProt peptide IDs that did not map to a fly gene symbol were excluded from analysis. Unsupervised clustering of samples with UMAP was performed using DEseq2 depth normalized LFQ values (Additional file 1: Figure S6).

\section{Analysis of differentially expressed transcripts and proteins}

Differential-expression analysis of transcripts and proteins was performed using DESeq2 [36]. As detailed above, for transcriptome analyses, elav $>\operatorname{Tau}^{W T}$ or elav $>\operatorname{Tau}^{R 406 W}$ were compared with the batch-matched elav control data (control set 1 or 2, respectively). For proteomic analyses, the single elav control set (control 3) was compared to either elav $>\mathrm{Tau}^{W T}$ or elav $>$ $\mathrm{Tau}^{R 406 \mathrm{~W}}$, and absolute peptide counts (LFQ) were used as the input for DESeq2 (which only accepts integers).
Raw transcript or peptide counts were normalized for library depth using DESeq2 median of ratios, and tested for differential expression using a generalized linear model. We initially determined Tau-induced differentially expressed transcripts or proteins cross-sectionally, examining Tau and control data separately at each time point (expression $\sim$ genotype, stratified by age for either 1-, 10-, or 20-day old animals). Subsequently, we performed joint regression analyses incorporating all longitudinal data, and including a covariate for age (expression $\sim$ genotype + age); the genotype term coefficient was used for significance testing. Age was used as a categorical, factor trait in our regression analyses to account for the possibility of non-linearity. Genes and proteins in Fig. 2 and Additional file 1: Figure S8 were plotted using log-transformed and depth-normalized expression or LFQ values. For determination of age-related changes in transcripts or proteins, our data was stratified by genotype, evaluating elav controls or elav > Tau flies separately, and age was used as the predictor variable. Differential expression was computed for either (i.) day 1 vs. day 10, (ii.) day 10 vs. day 20, or (iii.) day 1 vs. day 20. Significance testing was performed using the Wald test, implemented within DESeq2. In order to account for multiple-comparisons, the Benjamini-Hochberg procedure was applied, and a false discovery rate (FDR) < 0.05 was considered significant. In order to further evaluate the stringency of this FDR threshold, we applied a permutation procedure [37], in which genotype labels were shuffled within each experimental comparison and differential expression analysis was performed using DESeq2. Following 1000 permutations, we estimate an empirically-derived FDR corresponding to the $p$-value threshold selected from the experimental data (using the Benjamini-Hochberg procedure). For the proteomic studies of $\mathrm{Tau}^{\mathrm{R} 406 \mathrm{~W}}$ and $\mathrm{Tau}{ }^{\mathrm{WT}}$, this resulted in an empiric FDR estimate of 0.009 and 0.046 , respectively. For the transcriptome comparisons, the FDR estimates were 0.016 and 0.010 , respectively. As a sensitivity analysis to assess the potential impact of missing data and imputation on our proteomic analyses, we additionally examined age-adjusted differential protein expression in elav > Tau ${ }^{R 406 W}$ flies versus elav controls using 2 alternate datasets: (i) restricted to 1013 proteins with no missing values across all 27 samples or (ii) restricted to 2542 proteins with $<50 \%$ missingness. Full results from these analyses are included in Additional file 2: Table S12. All specific protein examples cited throughout the manuscript had complete, non-missing values in at least 2 out of 3 samples within each experimental group.

For analysis of concordance between transcriptome and proteome, we first examined the sign (positive or negative) of the genotype coefficient from the longitudinal (joint) regression model. Concordant 
transcripts were defined as having consistent direction of change (e.g. either positive or negative fold-change). We additionally implemented the survival $\mathrm{R}$ package [38], which calculates a concordance estimate using Somers' delta (D) in the formula (D+1)/2. Regression models were computed using the formula Transcript fold-change protein fold-change, considering (i) 1309 genes that were significantly differentially expressed in the transcriptome in response to $\mathrm{Tau}^{\mathrm{R} 406 \mathrm{~W}}$ or (ii) the subset of 261 genes for which both transcripts and proteins were differentially expressed. In this analysis, concordance estimates range from 0 (perfectly anti-correlated) to 1 (perfectly correlated), with 0.5 equating to random chance.

Functional enrichment for differentially expressed transcripts or proteins (joint regression model) was evaluated using the over-representation analysis (ORA) function of the WEB-based GEne SeT AnaLysis Toolkit [39]. All ORA analyses were conducted using the R implementation of WEBGESTALT. The minimum number of genes per category was set to 5 . We employed the following databases: GO biological processes, GO molecular functions, GO cellular component, KEGG, and Panther. Enrichment significance was defined using Fisher's exact test, followed by the Benjamini-Hochberg procedure; significance was set at $\mathrm{FDR}<0.05$.

\section{Hierarchical clustering and WGCNA analysis}

Hierarchical clustering was performed to evaluate $\mathrm{Tau}^{\mathrm{R} 406 \mathrm{~W}}$ associated, differentially-expressed transcripts $(n=4992$ genes), based on the joint regression model. Normalized expression counts for differentially-expressed genes were used as input. Pearson correlation was used as the distance metric and the complete linkage was used for distance calculation. Heatmaps of hierarchically clustered transcripts were generated using the heatmap.2 function from the gplots package in R. Based on a non-negative matrix factorization (NMF) rank survey using the NMF package in $\mathrm{R}$ [40], the optimal number of clusters was determined to be 6 , maximizing cophenetic scores while minimizing residuals (Additional file 1: Figure S12). This was applied to the clustering as a manual tree cut to yield 6 final clusters. Functional enrichment for cluster gene set was performed as described above. Concordance between transcripts in each cluster with corresponding proteins detected in the Tau ${ }^{\mathrm{R} 406 \mathrm{~W}}$ proteomic data was further evaluated by comparing the directions of $\log 2$ foldchanges. Median expression counts of genes belonging to each cluster were calculated from normalized expression values from all replicates in each genotype (Tau ${ }^{\mathrm{R} 406 \mathrm{~W}}$ or control set 2) and age.

Weighted gene coexpression network analysis (WGCNA) [41] was performed on expression counts from all $\mathrm{Tau}^{\mathrm{R}}{ }^{\mathrm{W} W \mathrm{~W}}$ transcripts $(n=10,217$ genes) after normalization in DESeq2 (median-of-ratios depth normalization). The soft threshold parameter was set at 5 , deepSplit $=4$, and minimum module size $=23$. Expression behavior of WGCNA modules were summarized by calculating module "eigengenes". Module eigengene is defined by PC1 loadings of a given module. Closely related modules were merged based on module eigengenes at a distance threshold of MEDissThres $=0.1$. The cluster dendrogram and module membership of transcripts are displayed in Additional file 1: Figure S13. Module eigengenes of each of the 15 resulting modules was examined for correlation with the $\mathrm{Tau}^{\mathrm{R} 406 \mathrm{~W}}$ genotype via Pearson correlation (Additional file 1: Figure S10). Normalized expression of genes in modules with module eigengenes that have significant correlation to the $\mathrm{Tau}^{\mathrm{R} 406 \mathrm{~W}}$ genotype were further evaluated in $\mathrm{Tau}^{\mathrm{R} 406 \mathrm{~W}}$ animals and controls (control set 2).

\section{Drosophila and human gene set overlaps}

In order to evaluate human-fly gene set overlaps, we first determined the fly homologs for all human $\mathrm{AD}$, tangle, or PSP differentially-expressed genes using the DRSC Integrated Ortholog Prediction Tool (DIOPT [42];), applying a minimum DIOPT score threshold of 5 (Additional file 2: Table S9). Where more than one fly homolog had a DIOPT score $>5$, all were included. We then computed enrichments of each human-derived data set (fly homologs) for either (i) Tau- or (ii) age-induced differentially expressed gene sets, based on our experimental analyses in Drosophila models, using the phyper base function of $\mathrm{R}$ to conduct a hypergeometric test. For Tau-induced fly genes, we include significant, differentially expressed genes from either the $\mathrm{Tau}^{R 406 W}$ or $\mathrm{Tau}^{W T}$ joint regression model (age-adjusted). For aging-induced fly genes, we considered all unique differentially expressed genes based on our analyses of elav control flies from multiple timepoints ( 1 vs. 10 days, 10 vs. 20 days, and 1 vs. 20 days), including from control sets 1 \& 2 for transcriptome studies or the complementary proteomic control set. For human-to-fly hypergeometric tests the overall background parameters for population size and population successes were based on the total number of unique and differentially-expressed genes in the Drosophila datasets: 17,104 and 5716, respectively, for the transcriptome, or 2742 and 548, respectively for the proteome. Other parameter values were as follows:

\begin{tabular}{lll}
\hline Human Data Set & Sample Size & Sample Successes \\
AD Transcriptome & 2426 & 1181 \\
PSP Transcriptome & 447 & 239 \\
Tangle Transcriptome & 1639 & 820 \\
AD Proteome & 524 & 63 \\
\hline
\end{tabular}

For the analyses integrating Drosophila RNA-seq and published modifiers, we again used DIOPT to determine 
the human homologs of relevant fly genes (DIOPT score $>5$ ).

\section{Validation of RNA-seq and LC-MS/MS}

For independent experimental validation of transcriptomic and proteomic findings, respectively, reverse transcription quantitative polymerase chain reaction (RT-qPCR) and western blots were performed following standard protocols as in prior published work [31]. For validation of RNA-seq, we examined the top $\mathrm{Tau}^{\mathrm{R} 406 \mathrm{~W}}$-induced differentially expressed transcripts $($ FDR $<0.05)$ at 10 days, including 10 up- and 10 downregulated transcripts, ranked based on high fold-change and low coefficient of variation $(\mathrm{CV}<0.3)$. PCR primers were selected using the FlyPrimerBank [43] (Additional file 1: Figure S4), and gene expression was normalized relative to $R p l 32$. For validation of LC/MS-MS, we considered 10 top-ranked gene expression changes in $\mathrm{Tau}^{\mathrm{R} 406 \mathrm{~W}}$ transgenic flies for which antibodies were available. We first ranked all significant results based on log fold change from the age-adjusted regression analysis restricted to non-missing proteins (Additional file 2: Table S12) and selected the top 50 up- and downregulated proteins. From this list of 100 proteins, we were able to identify and obtain antibodies for 7 targets: Mlp84B, Chic, Cp1, Gp93, Pdh, Futsch, and Chp. The specific timepoint for evaluation was selected based on the cross-sectional analysis (Additional file 2: Table S2). This candidate list was supplemented with 3 additional protein targets with missing data (Arf79F, Fln, Psc), based on a similar rank-list of the full imputed crosssectional analysis. Western blots were performed using the following antibodies and dilutions: rabbit antiMlp84B (1:200, from Dr. Mary Beckerle), mouse antichic (1:10, DSHB), mouse anti-Cp1/CTSL (clone 193, 702, MAB22591, 1:1000, R\&D Systems), rabbit antiGp93 (1:500, from Dr. Christopher Nicchitta), rabbit anti-Pdh (1:1000, from Dr. Craig Montell), mouse antifutsch (1:50, DSHB), mouse anti-chp (1:500, DSHB), mouse anti-fln (1:500, DSHB), mouse anti-Psc (1:5, DSHB), goat anti-Arf79F/ARF1 (ab58578, 1:250, Abcam), rabbit anti-GAPDH (1:2000, GeneTex), and mouse anti-Tubulin (DM1A, 1:1000, Sigma).

\section{Results}

Paired tau transcriptomes and proteomes in Drosophila Longitudinal, parallel RNA-seq and mass-spectrometry proteomics were performed in controls (elav-GAL4) and in flies with pan-neuronal expression of human wildtype $\left(\right.$ elav $\left.>T_{a u}{ }^{W T}\right)$ or mutant Tau $\left(e l a v>T_{a u}{ }^{R 406 W}\right)$. The transgenic genotypes and age timepoints (1-, 10-, and 20 -days) selected for this analysis have been extensively characterized in prior published work $[6,22]$, and we confirmed that $\mathrm{Tau}^{\mathrm{WT}}$ and $\mathrm{Tau}^{\mathrm{R} 406 \mathrm{~W}}$ are expressed at similar levels (Additional file 1: Figure S1). Overall, our quality-controlled and filtered datasets include 17,104 transcripts and 2723 proteins. We first analyzed our results cross-sectionally, highlighting those transcripts or proteins significantly differentially expressed (FDR $<$ 0.05 ) at each timepoint (Table 1; Additional file 2: Table S2). Overall, Tau ${ }^{\mathrm{WT}}$ altered expression of 1514 transcripts and 213 proteins. At each age examined, $\mathrm{Tau}^{\mathrm{R} 406 \mathrm{~W}}$ induced $\mathrm{a} \sim 4$ - to 7 -fold greater number of differentially expressed genes than Tau ${ }^{\mathrm{WT}}$, highlighting 5494 transcripts and 697 proteins. There was substantial overlap between the Tau ${ }^{\mathrm{WT}}$ and $\mathrm{Tau}^{\mathrm{R} 406 \mathrm{~W}}$ gene expression profiles, with $70 \%$ of $\mathrm{Tau}^{\mathrm{WT}}$-associated transcripts showing consistent changes in $\mathrm{Tau}^{\mathrm{R} 406 \mathrm{~W}}$ flies $(65 \%$ of proteins) (Additional file 1: Figure S2). Overall, nearly equal numbers of up- or down-regulated, differentially expressed genes were detected in the Tau transcriptome; whereas in the proteome, Tau-induced gene upregulation was more common by a factor of 2 , which may reflect reduced assay sensitivity for proteins with low expression levels (Additional file 1: Figure S3). Using RT-qPCR and western blots, we obtained consistent, independent experimental evidence to support validation of 15 out of 20 (75\%) differentially-expressed transcripts and 8 out of 10 proteins (80\%), systematically prioritized based on results from RNA-Seq and LC-MS/MS, respectively (Additional file 1: Figures S4 and S5).

As in human tauopathy, the neurodegenerative phenotypes manifested by Tau transgenic flies are progressive with aging [22]. Consistent with this, we observed age-dependent differences in the number and identity of differentially expressed genes across the timepoints examined (Additional file 1: Figure S2). For example, only a minority $(\sim 9 \%)$ of transcripts from Tau ${ }^{\text {WT }}$

Table 1 Tau-triggered differentially expressed genes

\begin{tabular}{lccccc}
\hline & Day 1 & Day 10 & Day 20 & \multicolumn{2}{c}{ Total } \\
\cline { 4 - 6 } & & & cross-sectional & age-adjusted \\
\hline Tau $^{\text {WT }}$ & $491(54)$ & $431(143)$ & $1096(76)$ & $1514(213)$ & $1653(123)$ \\
Tau $^{\text {R406W }}$ & $3179(97)$ & $1616(173)$ & $4087(581)$ & $5494(697)$ & $4992(503)$ \\
\hline
\end{tabular}

Differentially-expressed transcripts (and proteins, in parentheses) are indicated based on cross-sectional comparisons in 1-, 10-, or 20-day-old elav $>$ Tau ${ }^{\text {WT }}$ or elav > Tau ${ }^{R 406 W}$ animals and controls. Based on PCA analysis [31], the decrease in differentially expressed transcripts at day 10 in Tau ${ }^{R 406 W}$ flies is likely due to sample heterogeneity. The total number of unique differentially expressed transcripts/proteins from the cross-sectional analyses are also indicated, along with complementary results from the joint regression model including all longitudinal data and adjusting for age. Statistical analysis was based on a Wald test $($ FDR $<0.05)$. See Additional file 2: Tables S2 and S5 for complete results 
flies were consistently, differentially expressed at all 3 timepoints. The profound impact of aging on the Drosophila brain transcriptome and proteome is readily apparent in transcriptome-wide heatmaps (Fig. 1a,b) and unsupervised clustering analysis further highlights age as a major driver of gene expression differences among samples (Additional file 1: Figure S6). Indeed, among control animals, we documented age-related, differential expression of 6742 transcripts and 1155 proteins (Table 2 and Additional file 2: Tables S3, S4), and similar changes were seen in analyses of aged Tau animals (within genotype comparisons of data from different timepoints). Strikingly, approximately $70 \%$ of Tau-triggered transcripts overlap aging-associated gene expression changes. These data highlight an intimate connection between aging and Tau-mediated perturbations in gene expression.

\section{Integrated longitudinal analysis of differentially expressed transcripts and proteins}

In order to identify the most robust, Tau-induced expression changes independent of aging, we used linear regression and considered all longitudinal data in a joint model, including a covariate to adjust for age. In separate analyses of $\mathrm{Tau}^{\mathrm{WT}}$ and $\mathrm{Tau}^{\mathrm{R} 406 \mathrm{~W}}$, we identify 1653 and 4992 significant differentially expressed transcripts,

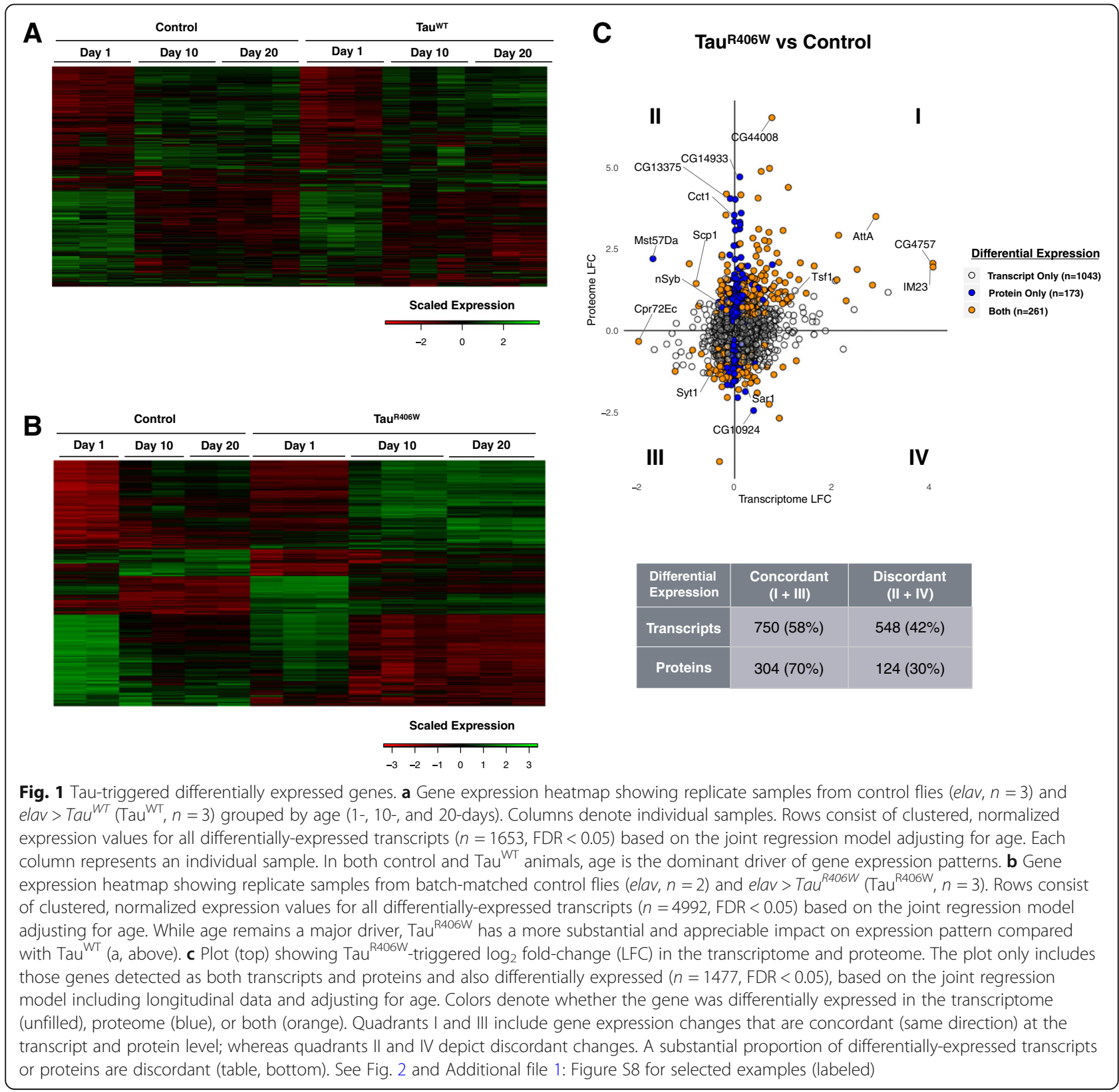


Table 2 Aging-triggered differentially expressed genes

\begin{tabular}{llll}
\hline & Control & Tau $^{\text {R406W }}$ & Change \\
\hline Transcripts & 6742 & 7970 & $+18 \%$ \\
Proteins & 1155 & 258 & $-78 \%$ \\
\hline In Tau & R406W animals, aging is associated with an increased number of \\
differentially-expressed transcripts but a decreased number of proteins. \\
Differentially-expressed transcripts and proteins were determined by \\
comparing aged animals, stratified by genotype, analyzing elav (control) or \\
elav $>$ Tau ${ }^{\text {R406W }}$ animals separately. The total number of unique, differentially- \\
expressed transcripts or proteins are shown based on the union of 3 \\
comparisons (1- vs. 10-days, 10- vs. 20-days, and 1- vs. 20-days). Statistical \\
analysis was based on a Wald test (FDR < 0.05). See Additional file 2: Tables S3 \\
and S4 for complete results
\end{tabular}

respectively (Table 1 and Additional file 2: Table S5). The same approach was applied to the longitudinal proteomic data. To better understand the joint impact of Tau on the transcriptome and proteome, we next examined those genes detected by both the RNA-seq and mass-spectrometry assays $\left(n=2395\right.$ and 2423 for Tau ${ }^{\text {WT }}$ and $\mathrm{Tau}^{\mathrm{R} 406 \mathrm{~W}}$, respectively). For this and subsequent analyses, we focus on the $\mathrm{Tau}^{\mathrm{R} 406 \mathrm{~W}}$ dataset given the more substantial number of differential expression changes (analyses of Tau ${ }^{\mathrm{WT}}$ are included as supplemental data and show consistent results). Remarkably, among $1309 \mathrm{Tau}^{\mathrm{R} 406 \mathrm{~W}}$-triggered, differentially expressed transcripts with corresponding proteome measurement, only $58 \%$ show concordant changes in the proteome (same direction of change, regardless of significance) (Fig. 1c and Additional file 1: Figure S7). These data indicate that for a substantial proportion of transcriptional changes (42\%), the behavior of corresponding proteins is discordant. Regression-based modeling of concordance (see Methods), incorporating both direction of effect and fold-change, confirmed that Tau-associated differential expression in the transcriptome is a weak but significant predictor of proteome behavior (estimate $=0.58, p=$ $\left.8.7 \times 10^{-5}\right)$. Since this analysis includes many transcripts with non-significant changes in the proteome, we also performed a more stringent, secondary analysis limited to 261 genes showing $\mathrm{Tau}^{\mathrm{R} 406 \mathrm{~W}}$-triggered, differential expression in both transcriptome and proteome. Among this subset, concordance was $77 \%$ based on consistent direction of change. While regression models confirmed increased concordance, there was no longer a significant relation between transcriptome and proteome behavior in this smaller sample (estimate $=0.65, p=0.18$ ). Consistent with these results, out of 503 significant, differentially expressed proteins induced by Tau ${ }^{\mathrm{R} 406 \mathrm{~W}}, 272(48 \%)$ are unique to the proteome (e.g. either non-significantly changed or not detected in the transcriptome).

Tau-mediated perturbations of the transcriptome and proteome are readily appreciated in an integrated plot (Fig. 1c) including all significant, differentially expressed transcripts and/or proteins, and representative examples discussed below are highlighted in Fig. 2 (see also
Additional file 1: Figure S8). Concordant activation or suppression of gene expression, respectively, is represented in the upper right (I) and lower left quadrants (III) of the plot. Many Tau-responsive genes show highly consistent and concordant expression changes in transcripts and proteins. Transferrin 1 (Tsf1) encoding an iron-binding protein induced during the Drosophila innate immune response [44] is strongly activated by $\mathrm{Tau}^{\mathrm{R} 406 \mathrm{~W}}$, showing similar $\sim 2$-fold increase in both the transcriptome and proteome, and these changes are largely consistent in 1-, 10-, and 20-day-old animals. Reciprocally, Synaptotagmin-1 (Syt1), encoding the essential calcium sensor for synaptic vesicle release and neurotransmission [45], is decreased $10 \%$ at the transcript level and $40 \%$ at the protein level, and this result agrees with prior targeted studies of synaptic proteins in $\mathrm{Tau}^{\mathrm{R} 406 \mathrm{~W}}$ flies [46]. By contrast, Tau-triggered gene expression changes that are discordant between the transcriptome and proteome occupy the upper left (II) and lower right (IV) quadrants of the plot (Fig. 1c). For example, Synaptobrevin (nSyb), which participates in synaptic vesicle fusion and release [47], is increased in Tau flies, whereas $n S y b$ transcripts are reciprocally decreased. Alternatively, in the case of Sar1, encoding a GTPase involved in endocytic trafficking [48], we detect a Tauassociated increase in transcripts, whereas Sar1 protein is decreased. Such discordant changes may suggest the possibility of feedback regulation between the transcriptome and proteome. In other cases, we detect significant Tau-induced changes in the proteome without a corresponding change in transcript levels. One such example is CCT1, encoding a cytosolic chaperone implicated in cytoskeletal regulation and nerve injury response [49].

As suggested above, aging has a profound impact on brain gene expression and frequently modifies the impact of Tau, sometimes with divergent consequences in the transcriptome and proteome. For example, aging is associated with a substantial increase in Tsf 1 transcript expression in both Tau and control animals ( 3- and 6-fold, respectively), whereas protein expression appears stable over the same timecourse. The immune response gene, Attacin-A (AttA), encoding an antimicrobial peptide, provides another striking example. RNA-seq reveals a consistent aging- and Tau-associated increase in AttA transcripts. However, the substantial Tau-associated increase observed in the proteome of 1day-old flies is attenuated during aging and no longer detected by 20-days (genotype $\mathrm{x}$ age interaction, $p=$ $3.78 \times 10^{-3}$ ). The sharp increase of AttA in wildtype flies with aging was previously reported and linked to neuronal maintenance [50]. Notably, in our age-adjusted joint model, only $35 \%$ of Tau-triggered differentially expressed transcripts were fully independent of aging. By contrast, the majority (65\%) were both Tau- and aging- 


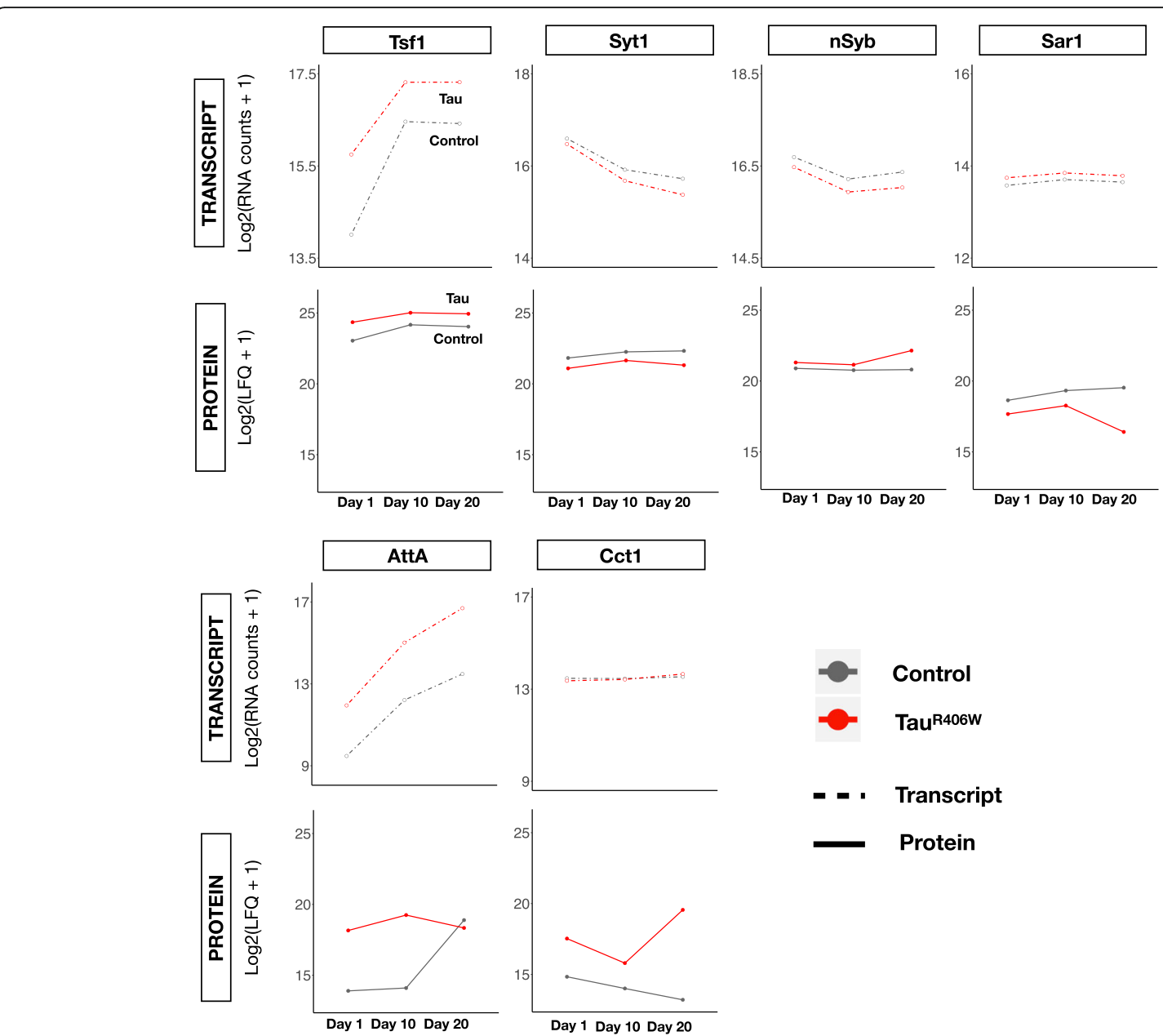

Fig. 2 Examples of Tau-induced changes in the transcriptome and proteome. $\log _{2}$-transformed expression of selected genes in elav $>$ Tau ${ }^{R 406 \mathrm{~W}}$ (Tau, red) and elav (Control, gray) is shown for transcriptome (depth normalized counts) and proteomes (normalized label-free quantification intensity (LFQ)). Genes were selected to be representative within our dataset and are all differentially expressed (FDR $<0.05)$ in both the transcriptome and proteome, based on the joint regression model including all longitudinal data and adjusting for age. CCT1 is only differentially-expressed at the protein level. AttA and CCT1 transcripts (bottom) are plotted on a different scale than the other examples due to the increased dynamic range of changes (for AttA). Additional example plots can be found in Additional file 1: Figure S8

associated gene expression changes (Additional file 1: Figure S9). Given the pervasive impact of aging, we again carefully considered all aging-associated changes, focusing on relative changes across the transcriptome and proteome, as well as a potential interaction with Taumediated toxicity (Table 2 and Additional file 2: Table S3, S4). Interestingly, in $\mathrm{Tau}^{\mathrm{R} 406 \mathrm{~W}}$ flies we note an $18 \%$ increase in aging-associated transcripts, with 7970 genes affected (versus 6742 in controls). However, within the proteome, the reverse pattern is seen with only 258 age-associated protein changes detected (versus 1155 in controls), representing a 78\% reduction, and potentially consistent with published reports of Tau-induced translational dysregulation [31, 51, 52] (see Discussion). A similar trend for the proteome is observed in $\mathrm{Tau}^{\mathrm{WT}}$ animals; although, the magnitude of changes was more modest (Additional file 2: Table S4). In sum, these data reveal unexpected and dynamic interactions between Tau and aging and their divergent impact on the transcriptome and proteome.

\section{Tau-induced gene expression networks implicate aging and innate immune pathways}

In order to reveal the broader biological processes disrupted by Tau, we next performed overrepresentation analysis using gene ontology (GO) annotations (Additional file 2: Table S6). We again focused on the $\mathrm{Tau}^{\mathrm{R} 406 \mathrm{~W}}$ age-adjusted dataset, given the greater number of differential expression changes, and we initially examined the transcriptome. Complementary analyses of $\mathrm{Tau}^{W T}$ are included in the supplemental data. Among all differentially expressed transcripts, we detected 
significant enrichment for genes implicated in synaptic function $\left(p=6.4 \times 10^{-35}\right)$, innate immunity $(p=3.4 \times$ $\left.10^{-14}\right)$, the cytoskeleton $\left(p=4.6 \times 10^{-11}\right)$, and endocytosis $\left(p=2.8 \times 10^{-9}\right)$. One potential limitation of this approach is that it considers the entire transcriptome as a single regulatory unit and may therefore be underpowered to detect more restricted network modules. Therefore, in order to partition the transcriptomic data into coregulated gene sets, we implemented unsupervised hierarchical clustering and defined 6 discrete gene sets ( $n=33-1863$ genes; Additional file 2: Table S7), equally divided between Tau-associated up- and down-regulated groups (Fig. 3). As expected, each cluster was significantly enriched for genes corresponding to the biological pathways outlined above (Additional file 2: Table S8), consistent with identification of discrete transcriptional regulatory networks. Four gene clusters revealed strong age-dependent changes in both control and Tau flies, including both age-dependent decreases (clusters 1 \& 3) or increases (clusters 2 \& 4). As expected, these clusters (1-4) strongly overlap with age-associated gene expression changes obtained from controls (mean $=78 \%$, range $66-85 \%$ overlap). Interestingly however, we observe 2 distinct patterns for the relationship between Tau- and aging-associated transcriptome changes. First, in gene sets enriched for immune (cluster 2, increasing with age) or synaptic biology (cluster 1 , decreasing with age), Tau amplifies the "aging expression signature". Conversely, in clusters 3 and 4-enriched for developmental and chromatin biology, respectively-Tau opposes the ageassociated changes. Thus, these 2 alternate patterns conform to accelerated versus delayed brain aging, based on the transcriptome responses. In contrast, neither of the remaining clusters reveal strong age-dependent changes in control flies, with Tau triggering decreased (cluster 5) or increased (cluster 6) gene expression. Interestingly, in

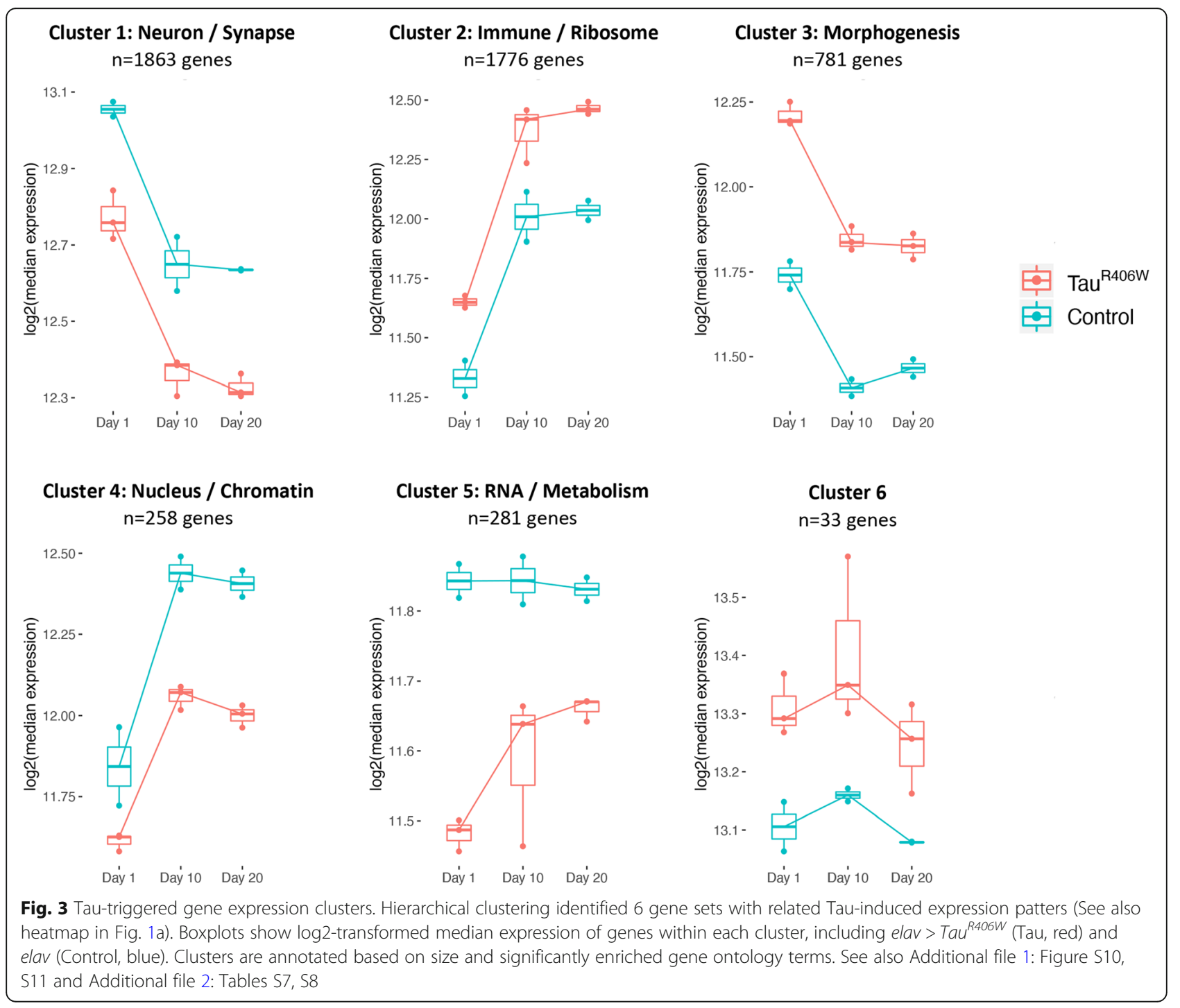


cluster 5, enriched for RNA and metabolic processes, the Tau-induced downregulation in the transcriptome appears to be attenuated by aging. As a complementary strategy to define Tau-associated gene regulatory networks, we also implemented weighted gene correlation network analysis (WGCNA), identifying 15 mutually exclusive transcriptional modules (Additional file 2: Table S7). Among these, we found 7 modules significantly associated with Tau genotype in $\mathrm{Tau}^{\mathrm{R} 406 \mathrm{~W}}$ flies (Additional file 1: Figure S10). Moreover, these modules substantially overlap with the gene sets defined using hierarchical clustering, resulting in similar functional enrichment profiles and recapitulating consistent interrelationships with aging (Additional file 2: Tables S7, S8).

In parallel analyses of the $\mathrm{Tau}^{\mathrm{R} 406 \mathrm{~W}}$ proteome dataset, we detected enrichment among differentially expressed proteins for translation $\left(p=3.1 \times 10^{-11}\right)$, including a preponderance of ribosomal proteins $\left(p=5.2 \times 10^{-19}\right)$ (Additional file 2: Table S6). We next integrated the transcriptome derived clusters with complementary data from proteomics. Consistent with our analyses described above, we found variable concordance among the clusters, based on the direction of differential expression detected in the proteome (Additional file 1: Figure S11). For example, clusters enriched for immune and synaptic function were reciprocally up- or down-regulated in Tau animals, but both gene sets were predominantly $(\sim 60 \%)$ concordant in the proteome-differentially expressed proteins show consistent direction of change in $\mathrm{Tau}^{\mathrm{R} 406 \mathrm{~W}}$ flies. By contrast, cluster 5, implicated in RNA processing and metabolism, showed only 39\% concordance, suggesting opposing regulatory interactions between the transcriptome and proteome.

\section{Cross-species annotation of tau-specific changes from human brain gene expression profiles}

Gene expression analysis from human postmortem brain tissue is confounded by mixed pathologies, making it difficult to identify those changes that are specifically triggered by Tau versus aging or other brain lesions. In contrast, our transcriptomic and proteomic analyses in flies benefit from matched experimental controls and longitudinal sampling, allowing definitive identification of Tau-triggered changes. We therefore leveraged our Drosophila results to annotate potential Tau-specific transcriptional changes from human brain gene expression profiles. We focused on 3 published analyses of differential gene expression, in relation to (i) AD clinicalpathologic diagnosis ( $n=478$ cases / 300 controls [10];), (ii) PSP clinical-pathologic diagnosis $(n=82$ cases / 76 controls [9];), or (iii) neurofibrillary tangle pathologic burden ( $n=478$ brains [11];). As expected, following homology mapping using the Drosophila Integrated Ortholog Prediction Tool [42]; 57-66\% of human genes had well-conserved fly homologs. The results of lookups are summarized in Table 3, and detailed results are included in Additional file 2: Table S9. In all 3 datasets, roughly half of conserved, differentially expressed changes are nominated as directly triggered by Tau pathology, based on cross-species annotation. Importantly, the observed human-fly overlaps appear more likely than that expected due to chance (hypergeometric test: $\mathrm{AD}, p=1.36 \times 10^{-63}$; PSP, $p=8.63 \times 10^{-19}$; tangle burden, $\left.p=1.81 \times 10^{-48}\right)$. Moreover, $\sim 50-60 \%$ of overlapping differentially expressed genes were concordant across species (i.e. gene up-regulation in both human $\mathrm{AD}$ and Drosophila Tau transcriptomes) (Additional file 2: Table S9). In a complementary analysis, we also examined the differentially expressed gene sets from human postmortem brains for overlaps with Drosophila aginginduced gene expression changes (Table 3 and Additional file 2: Table S9). An even greater proportion ( $70 \%$ ) of human genes altered in tauopathy showed conserved changes during brain aging in flies. In fact, few human genes specifically overlapped with the Tau dataset, with $90 \%$ overlapping both the Tau and aging differentially expressed gene sets. Lastly, we leveraged our fly proteomic data to annotate a recently reported massspectrometry dataset of differentially expressed proteins from 453 human brains, including $196 \mathrm{AD}$ clinicalpathologic cases and 257 controls [18]. Despite the reduced depth of coverage for proteomics, this additional analysis highlights 63 proteins differentially expressed in human $\mathrm{AD}$ for which fly protein homologs are similarly dysregulated in response to Tau; 471 proteins overlapped with the complementary fly aging-dysregulated proteins. Consistent results were obtained from an additional human tandem mass tag proteomics dataset [53]

Table 3 Tau- and aging-induced changes from cross-species overlaps

\begin{tabular}{lllll}
\hline Human & & & Drosophila & \\
\cline { 1 - 2 } Expression Dataset & Genes (Conserved) & & Tau (\%) & Aging (\%) \\
\hline AD & $3774(2426)$ & $1181(48.7 \%)$ & $1666(68.7 \%)$ \\
PSP & $745(447)$ & $239(53.5 \%)$ & $321(67.3 \%)$ \\
Tangles & $2485(1639)$ & $820(50.0 \%)$ & $1162(70.9 \%)$ \\
AD (proteins) & $959(524)$ & $63(12.0 \%)$ & $471(89.9 \%)$ \\
\hline
\end{tabular}

We examined differentially expressed transcripts from published RNA-seq analyses of human postmortem brain, including AD cases/controls [10], PSP cases/controls [9], or quantitative neurofibrillary tangle burden [11]. We also considered complementary mass-spectrometry proteomics from AD brains [18]. The total number of unique, differentially expressed human genes are noted along with the subset that are conserved in Drosophila. Among conserved genes, we examined the number and percentage with Tau- or aging-triggered differentially expressed homologs in flies. Given the reduced coverage of proteomics, we only consider conserved human proteins in which the homologous fly proteins were also detected in our assay. Overall consistent results were obtained from an additional human tandem mass tag proteomics dataset [53] including analyses of $18 \mathrm{AD}$ cases and 18 controls. For detailed results, see Additional file 2: Table S9 
including analyses of $18 \mathrm{AD}$ cases and 18 controls (Additional file 2: Table S9).

\section{Resolving amplifying versus protective expression changes using genetic modifiers}

Tau-associated gene expression changes are excellent candidates for causal mechanisms in tauopathies-those with the potential to alter disease onset, progression, and/or neurodegeneration (Fig. 4). Alternatively, differentially expressed genes may define non-causal perturbations-such changes may represent candidates as biomarkers for the neuronal injury accompanying neurofibrillary tangle pathology. Similar to recently published work [54], in order to identify potential causal gene expression changes, we integrated our findings with available results from 3 published, unbiased Drosophila screens, together defining 84 genetic modifiers of Taumediated neurotoxicity [23-25]. Among these, 37 genes were differentially expressed in the transcriptome and/or proteome (either $\mathrm{Tau}^{\mathrm{WT}}$ or $\mathrm{Tau}^{\mathrm{R} 406 \mathrm{~W}}$ ) (Table 4 and Additional file 2: Table S10). Next, for each of these 37 genes, we examined the direction of modifier tests from the literature to resolve whether the Tau-induced gene expression changes (up- or down-regulation) represent "amplifying" versus "protective" responses-that is, whether the observed perturbation in expression likely mediates or rather compensates for Tau-induced neuronal injury (Fig. 4). Up-regulated genes were defined as "amplifying" if genetic knockdown suppressed Tau toxicity and/or if overexpression reciprocally enhanced Tau phenotypes. For example, expression of Ubiquitin

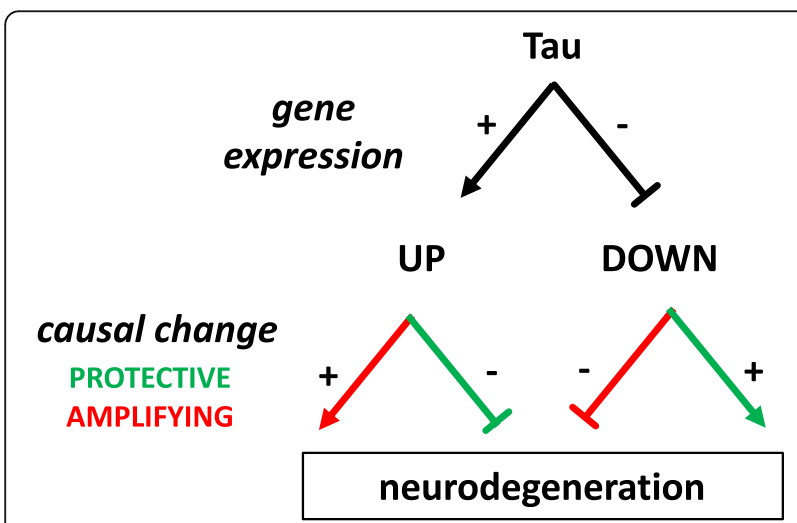

Fig. 4 Model for integrating Tau-induced gene expression changes and modifiers. Schematic diagram illustrating the relationship between Tau-induced perturbations in gene expression and potential impact on neurodegeneration. Tau may cause up- or down-regulation for a given gene of interest, and either change may amplify (red) or protect against (green) neurotoxicity. Recapitulating the observed gene expression change through experimental manipulations and observing the consequences for neurodegenerative phenotypes permits reconstruction of the causal chain. See Table 4 for specific examples
Table 4 Integration of gene expression with genetic modifiers

\begin{tabular}{|c|c|c|c|}
\hline \multicolumn{2}{|l|}{ Drosophila } & \multicolumn{2}{|l|}{ Human } \\
\hline Gene & $\begin{array}{l}\text { Amplifying vs. } \\
\text { Protective }\end{array}$ & $\overline{A D}$ & $\overline{\text { Tangles }}$ \\
\hline \multicolumn{4}{|c|}{ up-regulated } \\
\hline cher & A & & \\
\hline Ubal & A & & \\
\hline Myd88 & A & MYD88 & \\
\hline CG10889 & A & $\mathrm{ZC} 3 \mathrm{H} 12 \mathrm{C}$ & \\
\hline CG7970 & A & & \\
\hline Elf & A & GSPT1 & EIF2SI \\
\hline Fs(2)Ket & A & & \\
\hline Mekk1 & A & & \\
\hline $\mathrm{Nrg}$ & A & CHL1 & NRCAM \\
\hline smid & A & & \\
\hline Diap1 & A & BIRC3 & \\
\hline wun & A & PLPP1 & \\
\hline Stip1 & $P$ & STIP1 & \\
\hline RpS21 & $\mathrm{P}$ & RPS21 & \\
\hline Past1 & $\mathrm{P}$ & EHD2 & \\
\hline Tis 11 & $P$ & $\begin{array}{l}\text { ZFP36L1, } \\
\text { ZFP36L2 }\end{array}$ & \\
\hline$w$ & $P$ & & \\
\hline Gbs-70E & $P$ & PPP1R3C & \\
\hline cher & P & & \\
\hline dally & $P$ & & \\
\hline \multicolumn{4}{|c|}{ down-regulated } \\
\hline fry & A & & FRYL \\
\hline Ptp4E & A & & \\
\hline Atg6 & A & & \\
\hline Fmr1 & A & & \\
\hline mub & A & & \\
\hline Bacc & A & & \\
\hline jing & $P$ & & AEBP2 \\
\hline$E(b x)$ & $P$ & & BPTF \\
\hline tou & $P$ & & $B A Z 2 B$ \\
\hline jar & P & & \\
\hline$M i-2$ & $P$ & CHD5, CHD4 & CHD4 \\
\hline sgg & $P$ & & \\
\hline milt & $P$ & & TRAK2 \\
\hline stg & $P$ & $C D C 25 B$ & $C D C 25 B$ \\
\hline twe & $P$ & $C D C 25 B$ & $C D C 25 B$ \\
\hline At $\times 2$ & $P$ & & ATXN2L \\
\hline CG7231 & $P$ & & \\
\hline
\end{tabular}

All Drosophila genes listed (at left) are modifiers of Tau-mediated neurodegeneration based on published unbiased screens [23-25]. Direction of Tau-induced differential expression is noted, including up- (top) or downregulation (bottom) of transcripts. Based on the results of modifier tests, we can infer whether the observed Tau-induced expression perturbation is amplifying (A) or protective (P) for Tau neurotoxicity. See also Fig. 4 and Additional file 2: Table S9 and S10. For each fly gene, we also note whether human gene homolog(s) are differentially expressed in human postmortem brain tissue from published analyses of AD [10] and neurofibrillary tangle burden [11]. In cases where the direction of expression was concordant with Drosophila, the human gene name is indicated in boldface. In a smaller PSP dataset [9], only 1 fly gene, mub, had a differentially-expressed human homolog, PCBP4 
activating enzyme 1 (Uba1), a regulator of axon pruning, autophagy, and apoptosis [55-57], is significantly increased in $\mathrm{Tau}^{\mathrm{R} 406 \mathrm{~W}}$ flies. In published work [24], overexpression of Uba1 enhanced Tau-induced retinal degeneration, suggesting that the observed up-regulation likely promotes (amplifies) Tau toxicity. Conversely, expression of $\mathrm{Mi}-2$, encoding a CHD-family, chromatinremodeling enzyme, is significantly decreased in $\mathrm{Tau}^{\mathrm{R} 406 \mathrm{~W}}$ flies; however, since $\mathrm{Mi}-2$ is a loss-of-function suppressor of Tau neurotoxicity [25], we annotate this as a compensatory (protective) change. Interestingly, $U b a 1$ and $M i-2$ are members of expression clusters 2 \& 4, respectively (Fig. 3 and Additional file 2: Table S7), which are similarly characterized by age-associated upregulation but reveal opposing Tau-associated perturbations. Overall, we identify 18 amplifying (A) and 19 protective (P) gene expression changes induced by Tau. Thus, our transcriptome and proteome data can be integrated with genetic modifier studies to reconstruct a causal chain linking Tau, specific gene expression perturbations, and neurodegeneration. Moreover, 21 out of the 37 genes with published modifiers are also differentially expressed in one of the human datasets (Table 4 and Additional file 2: Table S9). For example, CHD5 (homolog of Mi-2) is decreased in human brains with $\mathrm{AD}$ pathology. Based on the complementary studies of fly $M i-2$, we can infer a potential Tau-triggered protective perturbation. These results demonstrate how Drosophila gene expression and genetic manipulation can be integrated to annotate human data for potential causal changes.

\section{Discussion}

Prior studies have profiled brain gene expression in Tau transgenic animals, including in flies $[29,30,58]$ and mouse models $[10,20,51]$; however, none to our knowledge have longitudinally assessed both transcripts and proteins in parallel. Our joint analyses therefore provide a glimpse of the dynamic regulatory crosstalk between the brain transcriptome and proteome accompanying brain injury, as in tauopathy. Remarkably, $42 \%$ of Tau-induced expression changes were discordant, with transcript and protein changing in opposite directions. This result is largely consistent with other emerging findings of surprisingly poor correlation between mRNA and protein levels among a variety of experimental systems $[16,59-62]$, including analyses of human postmortem brain tissue [17, 18, 63]. In one notable study relevant to $\mathrm{AD}$, a similar fraction $(40 \%)$ of differentially expressed transcripts in the 5XFAD amyloid precursor protein transgenic mouse showed discordant changes in the proteome [64]. Many discordant changes likely reflect regulatory feedback interactions that maintain protein homeostasis. Consistent with this, we found that transcript-protein concordance varied among coexpressed, and therefore likely coregulated, gene sets. Our longitudinal data also provides clues to primary perturbations in selected cases (e.g. Sar1 in Fig. 2); however, additional studies will be needed to confirm. Ultimately, successful translation from expression profiling studies requires unambiguous determination of whether a gene of interest is up- or down-regulated, but interpretation is currently limited by transcriptome-only analyses in most cases. Indeed, whether for nomination of potential therapeutic targets or development of diagnostic biomarkers, it will be essential to understand consequences at the protein level. We note that, among all differentially expressed transcripts, only a minority $(\sim 4 \%)$ were significantly and concordantly differentially expressed proteins--the remainder were either non-significantly differentially expressed, significant but discordant, or not detected at all by proteomics. Nevertheless, despite the comparatively reduced coverage of proteomics $(\sim 2700$ proteins vs. $\sim 17,000$ transcripts), many differentially expressed genes would not have been detected at all based on isolated transcriptional profiling. In the future, characterization of the proteome in Drosophila tauopathy models can be further enhanced by applying newer, more comprehensive methods (e.g. tandem mass tag labeling).

Age is the strongest known risk factor for $A D$, and aging-dependent progression is a defining feature of $\mathrm{AD}$ and other neurodegenerative tauopathies. As in studies of human postmortem tissue, most gene expression analyses of tauopathy models have been cross-sectional, partially obscuring the impact of aging and potential interactions with Tau-mediated changes. In our analyses, the majority $(\sim 70 \%)$ of Tau-triggered transcripts or proteins overlapped with those changes observed in aged control animals. Importantly, our longitudinal experimental design permitted identification of Tau-associated expression changes robust to aging adjustment. Remarkably however, even after adjustment, most Tau-mediated perturbations overlap with those seen in aging, and our cross-species analysis suggests consistent results for human tauopathy expression signatures. In short, our findings suggest that Tau pathology primarily modulates the endogenous gene expression programs of brain aging. Indeed, following hierarchical clustering, 4 out of 6 differentially expressed gene sets mirrored aging expression patterns, consistent with either Tau-accelerated or delayed aging. These complementary patterns may represent disease amplifying or protective responses, respectively, as shown for Uba1 and Mi-2. Interestingly, aging was associated with a quantitatively enhanced transcriptional signature in the Tau transgenic animals, characterized by an $18 \%$ increase in differentiallyexpressed genes. Reciprocally, Tau expression was accompanied by a $78 \%$ reduction in age-associated changes 
in the proteome. Though further investigation is warranted to confirm these observations, our analyses define Tau expression signatures in the both transcriptome and proteome enriched for genes implicated in translation, including numerous ribosomal proteins. Emerging evidence from both fly and mammalian animal models of tauopathy, as well as human postmortem tissue, suggest that Tau may be directly neurotoxic to transcription and translation. Specifically, pathologic forms of Tau interact with numerous RNA-binding protein factors comprising both the spliceosome and ribosome, and resulting disruptions of splicing and protein translation, respectively, likely contribute to Tau-mediated neurodegeneration $[31,51,52]$.

While aging has myriad systemic and cellular targets, one key emerging theme is the dysregulation of innate immune mechanisms leading to a systemic proinflammatory state, which has been termed "immunosenescence" or "inflamm-ageing" [65, 66]. In our analysis, innate immune pathways were strongly enriched among both aging- and Tau-associated, differentially expressed genes, and this result is consistent with brain gene expression profiling in mouse models of healthy aging [10, 67] and tauopathy $[68,69]$. Similarly, multiple transcriptome- and proteome-wide analyses of human postmortem brain from AD or other tauopathies, such as PSP, have identified evidence of dysregulated immune pathways $[9-11,18]$, and similar signatures have been implicated in brains from aged individuals without known neurodegenerative disease [70, 71]. Importantly, genome-wide association studies in $\mathrm{AD}$ highlight an abundance of susceptibility gene candidates implicated in immune regulation (e.g. TREM2, CD33, CR1), strongly suggesting a causal role in disease pathogenesis [72]. Further, polygenic modeling [73] and analyses of human cortical transcriptomes [74] converge to implicate activated microglia in the development of Tau pathology and susceptibility for AD. Numerous follow-up studies, including in mouse and cellular models, implicate microglia and astroglia with potential roles in propagating a pathogenic inflammatory cascade [75]. However, the prevailing mechanistic models of neuroinflammation in $\mathrm{AD}$ have largely focused on amyloid-beta as an upstream trigger and tau pathology as a downstream consequence, and the role of aging per se is often minimized. Nevertheless, primary tauopathies lacking amyloid pathology, such as PSP, and corresponding mouse models manifest prominent neuroinflammatory brain expression signatures. By contrast with mammals, neurons significantly outnumber glia in the Drosophila brain, and true microglial cells are not present in invertebrates [76]. Nevertheless, innate immune pathways are evolutionarily ancient, and toll-like receptor signaling components are not only expressed in fly neurons and glia, but they are required for brain maintenance in aging $[50,77]$. In the future, single-cell RNA-seq in Drosophila models of tauopathy may permit dissection of which cell types generate immune expression signatures along with complementary cell-type specific manipulations to confirm potential causal roles.

Gene expression profiling has emerged as a promising tool for functional genomic dissection of $\mathrm{AD}$ and other tauopathies; however, interpretation of these data can be powerfully enhanced by integration with complementary studies in model organisms. We have performed several cross-species analyses to highlight applications of our Drosophila tauopathy resource. One important challenge is to differentiate those gene expression changes specifically provoked by Tau-mediated mechanisms. Besides the influence of aging and life experiences, human brains commonly accumulate mixed pathologies [15]. By contrast, experimental models permit precisely-controlled manipulations that can isolate the responsible causal triggers. Roughly half of all conserved, differentiallyexpressed genes from the largest available analyses of human AD or PSP brain tissue were annotated as Tauinduced perturbations based on our Drosophila experiment. Remarkably, an even larger proportion of expression changes $(70 \%)$ were triggered by aging and we observed virtually complete overlap between Tau- and aging-associated changes. This result reinforces the intimate connection between the impact of neurodegenerative pathologies and aging on brain gene expression. Another major challenge following human gene expression analyses is to differentiate proximal causal pathways from more downstream, non-causal consequences of neurodegeneration. Experimental models permit controlled manipulations that mimic observed expression changes along with assessments to define potential impact on neurodegenerative phenotypes. In particular, Drosophila offers high-throughput genetics enabling unbiased, large-scale genetic screens for modifiers of Taumediated neurotoxicity [23-25]. By integrating these results with our RNA-seq findings, and cross-referencing with human gene expression profiles, we successfully highlight genes altered in human tauopathy that are strong candidates for further investigation as either amplifying or protective causal modifiers. In the future, targeted genetic manipulations of other conserved, differentiallyexpressed transcripts and/or proteins will significantly extend the value of our cross-species resource.

\section{Conclusions}

Our integrated, longitudinal analysis of the aging brain in Drosophila tauopathy models identifies perturbations affecting thousands of transcripts and hundreds of proteins and highlight many promising biological pathways. Among these, regulators of innate immunity, 
the cytoskeleton, endocytosis, and synaptic transmission, have independent support from genome-wide association studies of $\mathrm{AD}[72,78]$, neurofibrillary tangle burden [79], and PSP [80], consistent with causal roles. $\mathrm{Tau}^{\mathrm{R} 406 \mathrm{~W}}$, which causes familial FTD, was associated with a stronger impact on gene expression than Tau ${ }^{\mathrm{WT}}$, inducing up to 7-fold increased response in gene expression. While the overlap in differentially-expressed genes suggests shared mechanisms, our finding is consistent with both the enhanced neurotoxicity of $\mathrm{Tau}^{\mathrm{R} 406 \mathrm{~W}}$ in Drosophila [22, 81] and the more aggressive clinical profile of familial FTD (versus late-onset AD) [1, 2]. More broadly, we demonstrate pervasive overlap and reciprocal interactions between Tau- and aging-induced brain expression signatures as well as surprising discordance between transcripts and proteins. Lastly, cross-species analyses reveal extensive overlaps between Drosophila models and human postmortem brains with tauopathy. These results thus comprise a powerful, functional genomics resource for elucidation of Tau-mediated mechanisms of neurodegeneration.

\section{Supplementary information}

Supplementary information accompanies this paper at https://doi.org/10. 1186/s13024-020-00405-4.

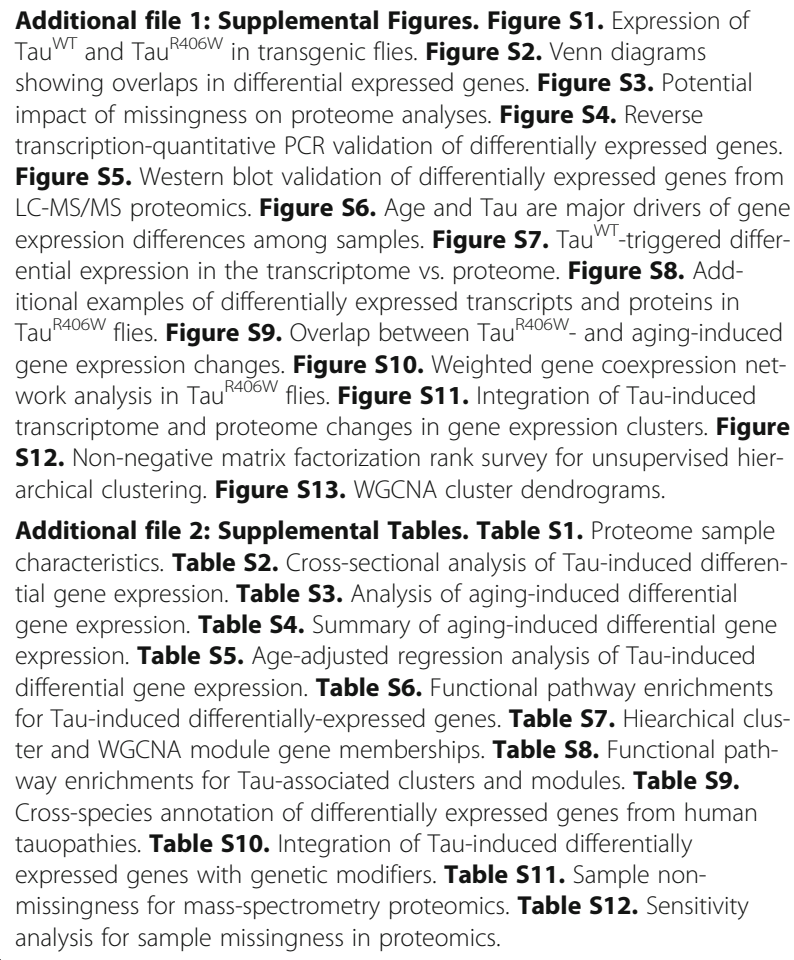

\section{Abbreviations}

MAPT: Microtubule Associated Protein Tau; AD: Alzheimer's disease; PSP: Progressive Supranuclear Palsy; FTD: Frontotemporal dementia; RNAseq: RNA-sequencing; GO: Gene ontology; WGCNA: Weighted gene correlation network analysis

\section{Acknowledgements}

We thank M.B. Feany for generously sharing Drosophila stocks and M.C. Beckerle, C.V. Nicchitta, C. Montell for antibody reagents.

\section{Authors' contributions}

Conceptualization, C.G.M., T.W., H.K.Y., N.T.S., Z.L., J.M.S.; Investigation and Analysis, C.G.M., T.W. H.K.Y., Y.-C.H., C.G., E.B.D., D.D.; Writing-Original Draft, C.G.M., T.W., H.K.Y., J.M.S.; Writing-Review \& Editing, C.G.M., T.W., H.K.Y., Y.C.H., C.G., D.D., E.B.D, P.L.D., N.T.S., J.M.S., Z.L.; Funding Acquisition, P.L.D., N.T.S., J.M.S., Z.L.; Supervision, N.T.S, J.M.S., Z.L. All authors have read and approved the final manuscript.

\section{Funding}

This study was supported by grants from the NIH (R01AG053960, R01AG050631, R01AG057339, U01AG061357, U01AG046161, R01AG057911, R01AG061800, RF1AG057471, RF1AG057470, R01AG061800, R01AG057911, R01AG057339). CMG was additionally supported by the Cullen Foundation, and both CMG and TW, by the Baylor College of Medicine Medical Scientist Training Program (MSTP). Z.L. received support from Cancer Prevention Research Institute of Texas RP170387, Houston Endowment, Huffington Foundation, and Belfer Neurodegenerative Disease Consortium. J.M.S. was additionally supported by Huffington Foundation, Jan and Dan Duncan Neurological Research Institute at Texas Children's Hospital, and a Career Award for Medical Scientists from the Burroughs Wellcome Fund.

\section{Availability of data and materials}

The complete RNAseq and proteomics data from Drosophila used in this study are available for download from the AMP-AD Knowledge Portal (www. synapse.org/ampad) (doi: https://doi.org/10.7303/syn7274101). Detailed results of our analyses are also included with this article and its additional files.

Ethics approval and consent to participate Not applicable.

\section{Consent for publication}

Not applicable.

\section{Competing interests}

The authors declare that they have no competing interests.

\section{Author details}

'Department of Molecular and Human Genetics, Baylor College of Medicine, Houston, TX 77030, USA. ${ }^{2}$ Medical Scientist Training Program, Baylor College of Medicine, Houston, TX 77030, USA. ${ }^{3}$ Department of Neuroscience, Baylor College of Medicine, Houston, TX 77030, USA. ${ }^{4}$ Department of Biochemistry, Emory University School of Medicine, Atlanta, GA 30322, USA. ${ }^{5}$ Center for Translational \& Computational Neuroimmunology, Department of Neurology and Taub Institute for the study of Alzheimer's disease and the aging brain, Columbia University Medical Center, New York, NY 10032, USA. ${ }^{6}$ Cell Circuits Program, Broad Institute, Cambridge, MA 02142, USA. 'Department of Neurology, Emory University School of Medicine, Atlanta, GA 30322, USA. ${ }^{8}$ Department of Pediatrics, Baylor College of Medicine, Houston, TX 77030, USA. ${ }^{9}$ Jan and Dan Duncan Neurological Research Institute, Texas Children's Hospital, 1250 Moursund St., Suite N.1150, Houston, TX 77030, USA.

${ }^{10}$ Department of Neurology, Baylor College of Medicine, Houston, TX 77030, USA.

Received: 29 February 2020 Accepted: 18 September 2020

Published online: 29 September 2020

\section{References}

1. Iqbal K, Liu F, Gong C-X. Tau and neurodegenerative disease: the story so far. Nat Rev Neurol. 2016;12(1):15-27.

2. Spillantini MG, Goedert M. Tau pathology and neurodegeneration. Lancet Neurol. 2013;12(6):609-22.

3. Hutton $\mathrm{M}$, Lendon $\mathrm{CL}$, Rizzu P, Baker M, Froelich $\mathrm{S}$, Houlden $\mathrm{H}$, et al. Association of missense and $5^{\prime}$-splice-site mutations in tau with the inherited dementia FTDP-17. Nature. 1998;393(6686):702-5.

4. Poorkaj P, Bird TD, Wijsman E, Nemens E, Garruto RM, Anderson L, et al. Tau is a candidate gene for chromosome 17 frontotemporal dementia. Ann Neurol. 1998;43(6):815-25. 
5. Spillantini MG, Murrell JR, Goedert M, Farlow MR, Klug A, Ghetti B. Mutation in the tau gene in familial multiple system tauopathy with presenile dementia. Proc Natl Acad Sci U S A. 1998;95(13):7737-41.

6. Gistelinck M, Lambert J-C, Callaerts P, Dermaut B, Dourlen P. Drosophila models of tauopathies: what have we learned? Int J Alzheimers Dis. 2012; 2012:970980.

7. Götz J, Bodea L-G, Goedert M. Rodent models for Alzheimer disease. Nat Rev Neurosci. 2018;19(10):583-98.

8. Wang $Y$, Mandelkow E. Tau in physiology and pathology. Nat Rev Neurosci. 2016:17(1):22-35.

9. Allen M, Wang X, Burgess JD, Watzlawik J, Serie DJ, Younkin CS, et al. Conserved brain myelination networks are altered in Alzheimer's and other neurodegenerative diseases. Alzheimers Dement. 2018;14(3):352-66.

10. Wan Y-W, Al-Ouran R, Mangleburg CG, Perumal TM, Lee TV, Allison K, et al Meta-analysis of the Alzheimer's disease human brain Transcriptome and functional dissection in mouse models. Cell Rep. 2020;32(2):107908.

11. Mostafavi S, Gaiteri C, Sullivan SE, White CC, Tasaki S, Xu J, et al. A molecular network of the aging human brain provides insights into the pathology and cognitive decline of Alzheimer's disease. Nat Neurosci. 2018;21(6):811-9.

12. Reynolds RH, Hardy J, Ryten M, Gagliano Taliun SA. Informing disease modelling with brain-relevant functional genomic annotations. Brain. 2019; 142(12):3694-712

13. Jack CR, Knopman DS, Jagust WJ, Petersen RC, Weiner MW, Aisen PS, et al. Tracking pathophysiological processes in Alzheimer's disease: an updated hypothetical model of dynamic biomarkers. Lancet Neurol. 2013;12(2):20716.

14. De Strooper B, Karran E. The cellular phase of Alzheimer's disease. Cell. 2016; 164(4):603-15.

15. Kapasi A, DeCarli C, Schneider JA. Impact of multiple pathologies on the threshold for clinically overt dementia. Acta Neuropathol. 2017;134(2):17186.

16. de Sousa Abreu R, Penalva LO, Marcotte EM, Vogel C. Global signatures of protein and mRNA expression levels. Mol BioSyst. 2009;5(12):1512-26.

17. Seyfried NT, Dammer EB, Swarup V, Nandakumar D, Duong DM, Yin L, et al. A Multi-network Approach Identifies Protein-Specific Co-expression in Asymptomatic and Symptomatic Alzheimer's Disease. Cell Syst. 2017;4(1): 60-72 e4.

18. Johnson ECB, Dammer EB, Duong DM, Ping L, Zhou M, Yin L, et al. Largescale proteomic analysis of Alzheimer's disease brain and cerebrospinal fluid reveals early changes in energy metabolism associated with microglia and astrocyte activation. Nat Med. 2020;26(5):769-80.

19. Gjoneska E, Pfenning AR, Mathys H, Quon G, Kundaje A, Tsai L-H, et al. Conserved epigenomic signals in mice and humans reveal immune basis of Alzheimer's disease. Nature. 2015;518(7539):365-9.

20. Wang H, Li Y, Ryder JW, Hole JT, Ebert PJ, Airey DC, et al. Genome-wide RNAseq study of the molecular mechanisms underlying microglia activation in response to pathological tau perturbation in the rTg4510 tau transgenic animal model. Mol Neurodegener. 2018;13(1):65.

21. Kim J, Selvaraji S, Kang SW, Lee WT, Chen CL-H, Choi H, et al. Cerebral transcriptome analysis reveals age-dependent progression of neuroinflammation in P301S mutant tau transgenic male mice. Brain Behav Immun. 2019;80:344-57.

22. Wittmann CW, Wszolek MF, Shulman JM, Salvaterra PM, Lewis J, Hutton M, et al. Tauopathy in Drosophila: neurodegeneration without neurofibrillary tangles. Science. 2001;293(5530):711-4.

23. Shulman JM, Feany MB. Genetic modifiers of tauopathy in Drosophila. Genetics. 2003;165(3):1233-42.

24. Blard O, Feuillette S, Bou J, Chaumette B, Frébourg T, Campion D, et al. Cytoskeleton proteins are modulators of mutant tau-induced neurodegeneration in Drosophila. Hum Mol Genet. 2007;16(5):555-66.

25. Ambegaokar SS, Jackson GR. Functional genomic screen and network analysis reveal novel modifiers of tauopathy dissociated from tau phosphorylation. Hum Mol Genet. 2011;20(24):4947-77

26. Shulman JM, Chipendo P, Chibnik LB, Aubin C, Tran D, Keenan BT, et al Functional screening of Alzheimer pathology genome-wide association signals in Drosophila. Am J Hum Genet. 2011:88(2):232-8.

27. Chapuis J, Hansmannel F, Gistelinck M, Mounier A, Van Cauwenberghe C, Kolen KV, et al. Increased expression of BIN1 mediates Alzheimer genetic risk by modulating tau pathology. Mol Psychiatry. 2013;18(11):1225-34.

28. Shulman JM, Imboywa S, Giagtzoglou N, Powers MP, Hu Y, Devenport D, et al. Functional screening in Drosophila identifies Alzheimer's disease susceptibility genes and implicates tau-mediated mechanisms. Hum Mol Genet. 2014;23(4):870-7.

29. Scherzer CR, Jensen RV, Gullans SR, Feany MB. Gene expression changes presage neurodegeneration in a Drosophila model of Parkinson's disease. Hum Mol Genet. 2003;12(19):2457-66.

30. Sekiya M, Wang M, Fujisaki N, Sakakibara Y, Quan X, Ehrlich ME, et al. Integrated biology approach reveals molecular and pathological interactions among Alzheimer's A 342 , tau, TREM2, and TYROBP in Drosophila models. Genome Med. 2018;10(1):26

31. Hsieh Y-C, Guo C, Yalamanchili HK, Abreha M, Al-Ouran R, Li Y, et al. TauMediated Disruption of the Spliceosome Triggers Cryptic RNA Splicing and Neurodegeneration in Alzheimer's Disease. Cell Rep. 2019;29(2):301-16 e10.

32. Dobin A, Davis CA, Schlesinger F, Drenkow J, Zaleski C, Jha S, et al. STAR: ultrafast universal RNA-seq aligner. Bioinformatics. 2013;29(1):15-21.

33. Becht E, Mclnnes L, Healy J, Dutertre C-A, Kwok IWH, Ng LG, et al. Dimensionality reduction for visualizing single-cell data using UMAP. Nat Biotechnol. 2019:37(1):38-44.

34. Lim MY, Paulo JA, Gygi SP. Evaluating false transfer rates from the matchbetween-runs algorithm with a two-proteome model. J Proteome Res. 2019;18(11):4020-6.

35. Tyanova S, Temu T, Sinitcyn P, Carlson A, Hein MY, Geiger T, et al. The Perseus computational platform for comprehensive analysis of (prote)omics data. Nat Methods. 2016;13(9):731-40.

36. Love MI, Huber W, Anders S. Moderated estimation of fold change and dispersion for RNA-seq data with DESeq2. Genome Biol. 2014;15(12):550.

37. Millstein J, Volfson D. Computationally efficient permutation-based confidence interval estimation for tail-area FDR. Front Genet. 2013;4:179.

38. Therneau TM, Grambsch PM. Modeling survival data: extending the cox model. New York: Springer-Verlag; 2000.

39. Liao Y, Wang J, Jaehnig EJ, Shi Z, Zhang B. WebGestalt 2019: gene set analysis toolkit with revamped UIs and APIs. Nucleic Acids Res. 2019;47(W1): W199-205.

40. Gaujoux R, Seoighe C. A flexible R package for nonnegative matrix factorization. BMC Bioinformatics. 2010;11(1):367.

41. Langfelder P, Horvath S. WGCNA: an R package for weighted correlation network analysis. BMC Bioinformatics. 2008;9(1):559.

42. Hu Y, Flockhart I, Vinayagam A, Bergwitz C, Berger B, Perrimon N, et al. An integrative approach to ortholog prediction for disease-focused and other functional studies. BMC Bioinformatics. 2011;12:357.

43. Hu Y, Sopko R, Foos M, Kelley C, Flockhart I, Ammeux N, et al. FlyPrimerBank: an online database for Drosophila melanogaster gene expression analysis and knockdown evaluation of RNAi reagents. G3: genes. Genomes Genetics. 2013;3(9):1607-16.

44. Yoshiga T, Georgieva T, Dunkov BC, Harizanova N, Ralchev K, Law JH. Drosophila melanogaster transferrin. Eur J Biochem. 1999;260(2):414-20.

45. Littleton JT, Stern M, Schulze K, Perin M, Bellen HJ. Mutational analysis of Drosophila synaptotagmin demonstrates its essential role in Ca2+-activated neurotransmitter release. Cell. 1993;74(6):1125-34.

46. Merlo P, Frost B, Peng S, Yang YJ, Park PJ, Feany M. p53 prevents neurodegeneration by regulating synaptic genes. PNAS. 2014;111(50):18055-60.

47. Broadie K, Prokop A, Bellen HJ, O'Kane CJ, Schulze KL, Sweeney ST. Syntaxin and synaptobrevin function downstream of vesicle docking in drosophila. Neuron. 1995;15(3):663-73.

48. Ivan V, de Voer G, Xanthakis D, Spoorendonk KM, Kondylis V, Rabouille C. Drosophila Sec16 mediates the biogenesis of tER sites upstream of Sar 1 through an arginine-rich motif. MBoC. 2008;19(10):4352-65.

49. Valakh V, Walker LJ, Skeath JB, DiAntonio A. Loss of the Spectraplakin short stop activates the DLK injury response pathway in Drosophila. J Neurosci. 2013;33(45):17863-73.

50. Kounatidis I, Chtarbanova S, Cao Y, Hayne M, Jayanth D, Ganetzky B, et al. NF-KB immunity in the brain determines Fly lifespan in healthy aging and age-related Neurodegeneration. Cell Rep. 2017;19(4):836-48.

51. Koren SA, Hamm MJ, Meier SE, Weiss BE, Nation GK, Chishti EA, et al. Tau drives translational selectivity by interacting with ribosomal proteins. Acta Neuropathol. 2019;137(4):571-83.

52. Meier S, Bell M, Lyons DN, Rodriguez-Rivera J, Ingram A, Fontaine SN, et al. Pathological tau promotes neuronal damage by impairing ribosomal function and decreasing protein synthesis. J Neurosci. 2016;36(3):1001-7.

53. Bai B, Wang X, Li Y, Chen P-C, Yu K, Dey KK, et al. Deep Multilayer Brain Proteomics Identifies Molecular Networks in Alzheimer's Disease Progression. Neuron. 2020;105(6):975-91 e7. 
54. Al-Ramahi I, Lu B, Paola SD, Pang K, de Haro M, Peluso I, et al. HighThroughput Functional Analysis Distinguishes Pathogenic, Nonpathogenic, and Compensatory Transcriptional Changes in Neurodegeneration. Cell Syst. 2018;7(1):28-40 e4

55. Chang T-K, Shravage BV, Hayes SD, Powers CM, Simin RT, Wade Harper J, et al. Uba1 functions in Atg7- and Atg3-independent autophagy. Nat Cell Biol. 2013;15(9):1067-78.

56. Lee TV, Ding T, Chen Z, Rajendran V, Scherr H, Lackey M, et al. The E1 ubiquitin-activating enzyme Uba1 in Drosophila controls apoptosis autonomously and tissue growth non-autonomously. Development. 2008; 135(1):43-52.

57. Watts RJ, Hoopfer ED, Luo L. Axon pruning during Drosophila metamorphosis: evidence for local degeneration and requirement of the ubiquitin-proteasome system. Neuron. 2003;38(6):871-85.

58. Sun W, Samimi H, Gamez M, Zare H, Frost B. Pathogenic tau-induced piRNA depletion promotes neuronal death through transposable element dysregulation in neurodegenerative tauopathies. Nat Neurosci. 2013;21(8):1038-48.

59. Aviner R, Shenoy A, Elroy-Stein O, Geiger T. Uncovering hidden layers of cell cycle regulation through integrative multi-omic analysis. PLoS Genet. 2015; 11(10):e1005554

60. Hartl D, Irmler M, Römer I, Mader MT, Mao L, Zabel C, et al. Transcriptome and proteome analysis of early embryonic mouse brain development. Proteomics. 2008:8(6):1257-65.

61. Lahtvee P-J, Sánchez BJ, Smialowska A, Kasvandik S, Elsemman IE, Gatto F, et al. Absolute Quantification of Protein and mRNA Abundances Demonstrate Variability in Gene-Specific Translation Efficiency in Yeast. Cell Syst. 2017;4(5):495-504 e5.

62. Zhang B, Wang J, Wang X, Zhu J, Liu Q, Shi Z, et al. Proteogenomic characterization of human colon and rectal cancer. Nature. 2014;513(7518): 382-7.

63. Robins C, Wingo AP, Fan W, Duong DM, Meigs J, Gerasimov ES, et al. Genetic control of the human brain proteome. bioRxiv. 2019;816652. https://doi.org/10.1101/816652.

64. Bundy JL, Vied C, Badger C, Nowakowski RS. Sex-biased hippocampal pathology in the 5XFAD mouse model of Alzheimer's disease: a multi-omic analysis. J Comp Neurol. 2019:527(2):462-75.

65. Franceschi C, Bonafè M, Valensin S, Olivieri F, Luca MD, Ottaviani E, et al. Inflamm-aging: an evolutionary perspective on Immunosenescence. Ann N Y Acad Sci. 2000;908(1):244-54

66. Shaw AC, Goldstein DR, Montgomery RR. Age-dependent dysregulation of innate immunity. Nat Rev Immunol. 2013;13(12):875-87.

67. Ximerakis M, Lipnick SL, Innes BT, Simmons SK, Adiconis X, Dionne D, et al. Single-cell transcriptomic profiling of the aging mouse brain. Nat Neurosci. 2019;22(10):1696-708.

68. Castanho I, Murray TK, Hannon E, Jeffries A, Walker E, Laing E, et al. Transcriptional Signatures of Tau and Amyloid Neuropathology. Cell Rep. 2020;30(6):2040-54 e5.

69. Matarin M, Salih DA, Yasvoina M, Cummings DM, Guelfi S, Liu W, et al. A genome-wide gene-expression analysis and database in transgenic mice during development of amyloid or tau pathology. Cell Rep. 2015;10(4):633-44.

70. Cribbs DH, Berchtold NC, Perreau V, Coleman PD, Rogers J, Tenner AJ, et al. Extensive innate immune gene activation accompanies brain aging, increasing vulnerability to cognitive decline and neurodegeneration: a microarray study. J Neuroinflammation. 2012;9(1):179.

71. Lu T, Pan Y, Kao S-Y, Li C, Kohane I, Chan J, et al. Gene regulation and DNA damage in the ageing human brain. Nature. 2004;429(6994):883-91.

72. Kunkle BW, Grenier-Boley B, Sims R, Bis JC, Damotte V, Naj AC, et al. Genetic meta-analysis of diagnosed Alzheimer's disease identifies new risk loci and implicates $A \beta$, tau, immunity and lipid processing. Nat Genet. 2019;51(3): 414-30.

73. Felsky D, Roostaei T, Nho K, Risacher SL, Bradshaw EM, Petyuk V, et al. Neuropathological correlates and genetic architecture of microglial activation in elderly human brain. Nat Commun. 2019;10(1):1-12.

74. Patrick $\mathrm{E}$, Olah $\mathrm{M}$, Taga $\mathrm{M}$, Klein $\mathrm{H}-\mathrm{U}, \mathrm{Xu}$ J, White CC, et al. A cortical immune network map identifies a subset of human microglia involved in Tau pathology. bioRxiv. 2017;234351. https://doi.org/10.1101/234351.

75. Heneka MT, Carson MJ, El Khoury J, Landreth GE, Brosseron F, Feinstein DL, et al. Neuroinflammation in Alzheimer's disease. Lancet Neurol. 2015;14(4): 388-405.

76. Yildirim K, Petri J, Kottmeier R, Klämbt C. Drosophila glia: few cell types and many conserved functions. Glia. 2019;67(1):5-26.
77. Davie K, Janssens J, Koldere D, Waegeneer MD, Pech U, Kreft $Ł$, et al. A Single-Cell Transcriptome Atlas of the Aging Drosophila Brain. Cell. 2018; 174(4):982-98 e20

78. Jansen IE, Savage JE, Watanabe K, Bryois J, Williams DM, Steinberg S, et al. Genome-wide meta-analysis identifies new loci and functional pathways influencing Alzheimer's disease risk. Nat Genet. 2019;51(3):404-13.

79. Chibnik LB, White CC, Mukherjee S, Raj T, Yu L, Larson EB, et al. Susceptibility to neurofibrillary tangles: role of the PTPRD locus and limited pleiotropy with other neuropathologies. Mol Psychiatry. 2018;23(6):1521-9.

80. Höglinger GU, Melhem NM, Dickson DW, Sleiman PMA, Wang L-S, Klei L, et al. Identification of common variants influencing risk of the tauopathy progressive supranuclear palsy. Nat Genet. 2011;43(7):699-705.

81. Bardai FH, Wang L, Mutreja Y, Yenjerla M, Gamblin TC, Feany MB. A conserved cytoskeletal signaling Cascade mediates neurotoxicity of FTDP-17 tau mutations in vivo. J Neurosci. 2018:38(1):108-19.

\section{Publisher's Note}

Springer Nature remains neutral with regard to jurisdictional claims in published maps and institutional affiliations.

Ready to submit your research? Choose BMC and benefit from:

- fast, convenient online submission

- thorough peer review by experienced researchers in your field

- rapid publication on acceptance

- support for research data, including large and complex data types

- gold Open Access which fosters wider collaboration and increased citations

- maximum visibility for your research: over $100 \mathrm{M}$ website views per year

At BMC, research is always in progress.

Learn more biomedcentral.com/submissions 